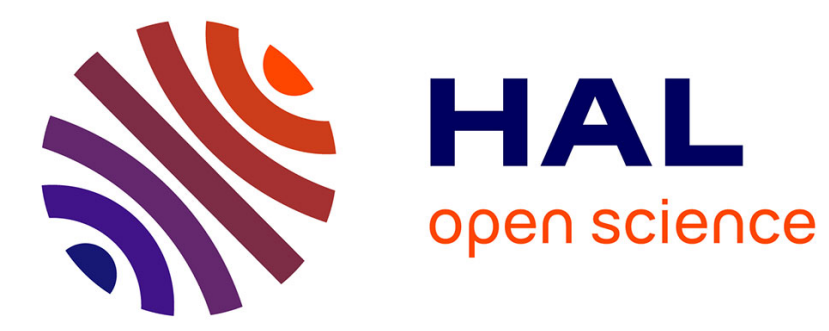

\title{
Robustness of Liouville Measure under a Family of Stable Diffusions
}

Francois Ledrappier, Lin Shu

\section{To cite this version:}

Francois Ledrappier, Lin Shu. Robustness of Liouville Measure under a Family of Stable Diffusions. Communications on Pure and Applied Mathematics, 2020, 73 (12), pp.2708-2736. 10.1002/cpa.21935 . hal-03095571

\section{HAL Id: hal-03095571 \\ https://hal.sorbonne-universite.fr/hal-03095571}

Submitted on 4 Jan 2021

HAL is a multi-disciplinary open access archive for the deposit and dissemination of scientific research documents, whether they are published or not. The documents may come from teaching and research institutions in France or abroad, or from public or private research centers.
L'archive ouverte pluridisciplinaire HAL, est destinée au dépôt et à la diffusion de documents scientifiques de niveau recherche, publiés ou non, émanant des établissements d'enseignement et de recherche français ou étrangers, des laboratoires publics ou privés. 


\title{
A FAMILY OF STABLE DIFFUSIONS
}

\author{
FRANÇOIS LEDRAPPIER AND LIN SHU
}

\begin{abstract}
Consider a $C^{\infty}$ closed connected Riemannian manifold $(M, g)$ with negative curvature. The unit tangent bundle $S M$ is foliated by the (weak) stable foliation $\mathcal{W}^{s}$ of the geodesic flow. Let $\Delta^{s}$ be the leafwise Laplacian for $\mathcal{W}^{s}$ and let $\bar{X}$ be the geodesic spray, i.e., the vector field that generates the geodesic flow. For each $\rho$, the operator $\mathcal{L}_{\rho}:=\Delta^{s}+\rho \bar{X}$ generates a diffusion for $\mathcal{W}^{s}$. We show that, as $\rho \rightarrow-\infty$, the unique stationary probability measure for the leafwise diffusion of $\mathcal{L}_{\rho}$ converge to the normalized Liouville measure on $S M$.
\end{abstract}

\section{Statement of the Result}

Let $(M, g)$ be an $m$-dimensional closed connected negatively curved $C^{\infty}$ Riemannian manifold. We shall study a class of probability measures on the unit tangent bundle $S M$ which interpolates between the Burger-Roblin measure (whose transversal distribution in the weak unstable leaves is the same as the one for the maximal entropy measure of the geodesic flow) and the normalized Liouville measure.

Let $\widetilde{g}$ be the the $G$-invariant extension of $g$ to the universal cover space $\widetilde{M}$. The fundamental group $G=\pi_{1}(M)$ acts on $(\widetilde{M}, \widetilde{g})$ as isometries such that $M=\widetilde{M} / G$. Let $\partial \widetilde{M}$ be the geometric boundary of $\widetilde{M}$, i.e., the collection of equivalent classes of unit speed geodesic rays that remain a bounded distance apart. Since $\widetilde{g}$ is negatively curved, there is a natural homeomorphism from $\partial \widetilde{M}$ to the unit sphere $S_{x} \widetilde{M}$ in the tangent space at $x \in \widetilde{M}$, sending $\xi$ to the initial vector of the geodesic ray starting from $x$ in the equivalent class of $\xi([$ EO73 $])$. Hence we identify the unit tangent bundle $S \widetilde{M}=\bigcup_{x \in \widetilde{M}} S_{x} \widetilde{M}$ with $\widetilde{M} \times \partial \widetilde{M}$.

For each $\mathbf{v}=(x, \xi) \in S \widetilde{M}$, its (weak) stable manifold for the geodesic flow $\left\{\boldsymbol{\Phi}_{t}\right\}_{t \in \mathbb{R}}$ on $S \widetilde{M}$, denoted $\widetilde{W}^{s}(\mathbf{v})$, is the collection of initial vectors of geodesic rays in the equivalent class of $\xi$ and can be identified with $\widetilde{M} \times\{\xi\}$. The collection of $\widetilde{W}^{s}(\mathbf{v})$ form the stable foliation $\widetilde{\mathcal{W}}^{s}$ of $S \widetilde{M}$. Extend the action of $G$ continuously to $\partial \widetilde{M}$. Then $S M$ can be identified with the quotient of $\widetilde{M} \times \partial \widetilde{M}$ under the diagonal action of $G$. Since $\psi\left(\widetilde{W}^{s}(\mathbf{v})\right)=$ $\widetilde{W}^{s}(D \psi(\mathbf{v}))$ for $\psi \in G$, the collection of quotients of $\widetilde{W}^{s}(\mathbf{v})$ defines a lamination $\mathcal{W}^{s}$ on $S M$, the so-called (weak) stable foliation of $S M$. The leaves of $\mathcal{W}^{s}$ are discrete quotients of

2010 Mathematics Subject Classification. 37D40, 58J65.

Key words and phrases. Harmonic measure, Liouville measure.

The second author was partially supported by NSFC (No.11331007 and No.11422104). 
$\widetilde{M}$, which are naturally endowed with the Riemannian metric induced from $\widetilde{g}$. For $v \in S M$, let $W^{s}(v)$ be the leaf of $\mathcal{W}$ containing $v$. Then $W^{s}(v)$ is a $C^{\infty}$ immersed submanifold of $S M$ depending Hölder continuously on $v$ in the $C^{\infty}$-topology ([Shu87]).

Let $\mathcal{L}$ be a Markovian operator (i.e., $\mathcal{L} 1=0$ ) on (the smooth functions on) $S M$ with continuous coefficients. It is said to be subordinated to the stable foliation $\mathcal{W}^{s}$, if for every smooth function $f$ on $S M$, the value of $\mathcal{L}(f)$ at $v \in S M$ only depends on the restriction of $f$ to $W^{s}(v)$. A Borel probability measure $\mathbf{m}$ on $S M$ is called $\mathcal{L}$-harmonic if it satisfies

$$
\int \mathcal{L}(f) d \mathbf{m}=0
$$

for every smooth function $f$ on $S M$. Extend $\mathcal{L}$ to be a $G$-equivariant operator on $S \widetilde{M}=$ $\widetilde{M} \times \partial \widetilde{M}$, which we shall denote with the same symbol, and, for $\mathbf{v}=(x, \xi) \in S \widetilde{M}$, let $\mathcal{L}^{\mathbf{v}}$ denote the laminated operator of $\mathcal{L}$ on $\widetilde{W}^{s}(\mathbf{v})=\widetilde{M} \times\{\xi\}$. Call $\mathcal{L}$ weakly coercive, if its lifted leafwise operators $\mathcal{L}^{\mathbf{v}}, \mathbf{v} \in S \widetilde{M}$, are weakly coercive in the sense that there are a number $\varepsilon>0$ (independent of $\mathbf{v}$ ) and, for each $\mathbf{v}$, a positive $\left(\mathcal{L}^{\mathbf{v}}+\varepsilon\right)$-superharmonic function $F$ on $\widetilde{M}$ (i.e., $\left.\left(\mathcal{L}^{\mathbf{v}}+\varepsilon\right) F \leqslant 0\right)$. It is known that for a weakly coercive operator, there exists a unique harmonic measure ([Gar83], [Ham97]).

One classical example of weakly coercive operator is $\mathcal{L}=\Delta^{s}$, the laminated Laplacian for $\mathcal{W}^{s}$, whose unique $\mathcal{L}$-harmonic measure is always referred to as the harmonic measure ([Gar83]). Many interesting open problems in dynamics are concerned with the relationship of the harmonic measure with the normalized Liouville measure and the normalized maximal entropy measure for the geodesic flow (Bowen-Margulis measure), and the applications of these relations to the characterizations of the locally symmetric property of the underlying space (see [Kat82, Sul83] and see also [Kai90, Led95, Yue94] for more descriptions).

In this paper, we are interested in the family $\mathcal{L}_{\rho}=\Delta^{s}+\rho \bar{X}$, where $\rho$ is a real number and $\bar{X}$ is the geodesic spray. Since $\bar{X}$ is tangent to the stable manifold, the operators $\mathcal{L}_{\rho}$ are subordinated to the stable foliation.

Let $V$ denote the volume entropy of $(M, g)$ :

$$
V=\lim _{r \rightarrow+\infty} \frac{\log \operatorname{Vol}(B(x, r))}{r}
$$

where $B(x, r)$ is the ball of radius $r$ in $(\widetilde{M}, \widetilde{g})$ and Vol is the volume. The volume entropy coincides with the topological entropy of the geodesic flow on $S M$ since $g$ has negative sectional curvature ([Man79]). For $\rho<V$, the operator $\mathcal{L}_{\rho}$ is weakly coercive ([Ham97]) and hence there is a unique $\mathcal{L}_{\rho}$-harmonic measure, which we will denote by $\mathbf{m}_{\rho}$.

Clearly, $\mathbf{m}_{0}$ is the classical harmonic measure. When $\rho \rightarrow V, \mathbf{m}_{\rho}$ tends to the BurgerRoblin measure $\mathbf{m}_{B R}$, the unique harmonic measure for the Laplacian subordinated to the strong stable foliation ([LS, Proposition 4.10], the uniqueness of such a measure is due to Kaimanovich $([$ Kai88 $]))$. When $\rho \rightarrow-\infty$, the main result of this paper is: 
Theorem 1.1. Let $(M, g)$ be an m-dimensional closed connected negatively curved $C^{\infty}$ Riemannian manifold. As $\rho \rightarrow-\infty$, the $\mathcal{L}_{\rho}$-harmonic measure $\mathbf{m}_{\rho}$ converge to the normalized Liouville measure on $S M$.

Roughly speaking, since the measure $\mathbf{m}_{\rho}$ is $\mathcal{L}_{\rho^{-}}$-harmonic, it is also stationary for the operator $-\bar{X}-(1 / \rho) \Delta^{s}$ (see Section 2 for a precise definition). In particular, any limit measure of the family $\mathbf{m}_{\rho}$ as $-1 / \rho \rightarrow 0$ is invariant under the (reversed) geodesic flow. For a limit of random perturbations of a conservative Anosov flow, the convergence of the stationary measures to a SRB measure has been shown by several authors, in particular Kifer ([Kif74] $)$, under the condition that the operator $\Delta$ is hypoelliptic, so that the Markov kernels have a density with respect to Lebesgue on $S M$. We cannot apply this to show Theorem 1.1 since in our case, the operators are subordinated to the stable foliation and the Markov kernels $\mathbf{p}_{\rho}(t,(x, \xi), d(y, \eta))$ are singular. Another approach by Cowieson-Young ([CY05]) uses the variational principle from thermodynamical formalism and we show that such an approach can be used in our case in spite of the singularity of the Markov kernels. We shall show any limiting measure $\mathbf{m}$ of $\mathbf{m}_{\rho}$ (as $\left.\rho \rightarrow-\infty\right)$ satisfies Pesin entropy formula for the geodesic flow. Theorem 1.1 follows since the normalized Liouville measure on $S M$ is indeed characterized by Pesin formula among invariant measures for the geodesic flow ([BR75]). More precisely, we will define a stochastic flow on a bigger space and consider a special stationary measure $\overline{\mathbf{m}}_{\rho}$ for that stochastic flow that projects to $\mathbf{m}_{\rho}$ on $S M$. We then introduce a relative entropy like quantity $h_{\rho}^{s}$ for $\overline{\mathbf{m}}_{\rho}$ and show $h_{\mathbf{m}}$, the entropy of $\mathbf{m}$ for the reversed geodesic flow, satisfies

$$
h_{\mathbf{m}} \geqslant \limsup _{\rho \rightarrow-\infty} h_{\rho}^{s}
$$

This can be done (see Proposition 3.2) along the lines of Cowieson-Young ([CY05]) and Kifer-Yomdin ([KY 88 $]$ ) for the upper semi-continuity of the relative entropy. To conclude Theorem 1.1, we verify that $\lim \sup _{\rho \rightarrow-\infty} h_{\rho}^{s}$ has a lower bound given by Pesin entropy integral for $\mathbf{m}$ using the SRB like properties of $\overline{\mathbf{m}}_{\rho}$ (see Proposition 3.1) and their nice convergence property inherited from our stochastic flow system (see Proposition 2.7).

We arrange the paper as follows. In Section 2, we will give preliminaries on the properties of the $\mathcal{L}$-harmonic measures and the dynamics of the associated stochastic flows. In Section 3 , we will introduce the random system to define $h_{\rho}^{s}$ and reveal its relation with Pesin entropy formula. The upper semi-continuity equality (1.1) will be shown in the final section.

\section{HARMONiC MEASURE AND STOCHASTIC FLOW}

We begin with some basic understanding of the $\mathcal{L}_{\rho}$-harmonic measure $\mathbf{m}_{\rho}(\rho<V)$ by analyzing the dynamics of its $G$-invariant extension on $\widetilde{M} \times \partial \widetilde{M}$, which is denoted by $\widetilde{\mathbf{m}}_{\rho}$.

Consider the $G$-equivariant extension of $\mathcal{L}_{\rho}$ to $S \widetilde{M}=\widetilde{M} \times \partial \widetilde{M}$, which we shall denote by the same symbol. It defines a Markovian family of probabilities on $\widetilde{\Omega}_{+}$, the space of paths of $\widetilde{\omega}: \mathbb{R}_{+} \rightarrow S \widetilde{M}$ (where $\mathbb{R}_{+}:=[0,+\infty)$ ), equipped with the smallest $\sigma$-algebra $\mathcal{A}$ 
for which the projections $R_{t}: \widetilde{\omega} \mapsto \widetilde{\omega}(t)$ are measurable. Indeed, for $\mathbf{v}=(x, \xi) \in S \widetilde{M}$, the laminated operator $\mathcal{L}_{\rho}^{\mathbf{v}}$ on $\widetilde{W}^{s}(\mathbf{v})$ can be regarded as an operator on $\widetilde{M}$ with corresponding heat kernel functions $p_{\rho}^{\mathbf{v}}(t, y, z), t>0, y, z \in \widetilde{M}$. Define

$$
\mathbf{p}_{\rho}(t,(x, \xi), d(y, \eta))=p_{\rho}^{\mathbf{v}}(t, x, y) d \operatorname{Vol}(y) \delta_{\xi}(\eta),
$$

where $\delta_{\xi}(\cdot)$ is the Dirac function at $\xi$. Then the diffusion process on $\widetilde{W}^{s}(\mathbf{v})$ with infinitesimal operator $\mathcal{L}_{\rho}^{\mathbf{v}}$ is given by a Markovian family $\left\{\mathbb{P}_{\rho}^{\mathbf{w}}\right\}_{\mathbf{w} \in \widetilde{M} \times\{\xi\}}$, where for every $t>0$ and every Borel set $A \subset \widetilde{M} \times \partial \widetilde{M}$ we have

$$
\mathbb{P}_{\rho}^{\mathbf{w}}(\{\widetilde{\omega}: \widetilde{\omega}(t) \in A\})=\int_{A} \mathbf{p}_{\rho}(t, \mathbf{w}, d(y, \eta)) .
$$

Proposition 2.1. ([Gar83, Ham97]) With the above notations, the following are true for $\rho<V$.

i) The measure $\widetilde{\mathbf{m}}_{\rho}$ satisfies, for all $f \in C^{2}(\widetilde{M} \times \partial \widetilde{M})$ with compact support,

$$
\int_{\widetilde{M} \times \partial \widetilde{M}}\left(\int_{\widetilde{M} \times \partial \widetilde{M}} f(y, \eta) \mathbf{p}_{\rho}(t,(x, \xi), d(y, \eta))\right) d \widetilde{\mathbf{m}}_{\rho}(x, \xi)=\int_{\widetilde{M} \times \partial \widetilde{M}} f(x, \xi) d \widetilde{\mathbf{m}}_{\rho}(x, \xi) .
$$

ii) The measure $\widetilde{\mathbb{P}}_{\rho}=\int \mathbb{P}_{\rho}^{\mathbf{v}} d \widetilde{\mathbf{m}}_{\rho}(\mathbf{v})$ on $\widetilde{\Omega}_{+}$is invariant under all the shift maps $\left\{\sigma_{t}\right\}_{t \in \mathbb{R}_{+}}$ on $\widetilde{\Omega}_{+}$, where $\sigma_{t}(\widetilde{\omega}(s))=\widetilde{\omega}(s+t)$ for $s \in \mathbb{R}_{+}$and $\widetilde{\omega} \in \widetilde{\Omega}_{+}$.

iii) The measure $\widetilde{\mathbf{m}}_{\rho}$ can be expressed locally at $\mathbf{v}=(x, \xi) \in S \widetilde{M}$ as $d \widetilde{\mathbf{m}}_{\rho}=k_{\rho}(y, \eta)(d y \times$ $\left.d \nu_{\rho}(\eta)\right)$, where $\nu_{\rho}$ is a finite measure on $\partial \widetilde{M}$ without atoms and, for $\nu_{\rho}$-almost every $\eta, k_{\rho}(y, \eta)$ is a positive function on $\widetilde{M}$ satisfying the equation

$$
\Delta\left(k_{\rho}(y, \eta)\right)-\rho \operatorname{Div}\left(k_{\rho}(y, \eta) \bar{X}(y, \eta)\right)=0,
$$

where we continue to use $\bar{X}$ to denote the geodesic spray for $S \widetilde{M}$.

Remark 2.2. Let $\mathbf{m}$ be any weak* limit of the probability measures $\mathbf{m}_{\rho}$ on $S M$ as $\rho \rightarrow-\infty$ and let $\widetilde{\mathbf{m}}$ be the $G$-invariant extension of $\mathbf{m}$ to $\widetilde{M} \times \partial \widetilde{M}$. Clearly, Theorem 1.1 follows if we can show $\widetilde{\mathbf{m}}$ has absolutely continuous conditional measures on leafs $\widetilde{M} \times\{\xi\}$. But this does not follow directly from equation (2.1) since the Harnack inequality used for each $\rho$ finite is worse and worse when $\rho$ goes to $-\infty$ and hence we have less and less control of the density functions $k_{\rho}$.

For Theorem 1.1, we will further explore the invariant dynamics of $\mathbf{m}_{\rho}$ from the stochastic flow point of view and use it to establish the entropy formula for the limit measures.

We first recall some classical results from the theory of Stochastic Differential Equations (SDE). Let $\left\{B_{t}=\left(B_{t}^{1}, \cdots, B_{t}^{d}\right)\right\}_{t \in \mathbb{R}_{+}}$be a $d$-dimensional Euclidean Brownian motion starting from the origin with the Euclidean Laplacian generator (so the covariance matrix is $2 t \mathrm{Id})$ and let $(\Omega, \mathbb{P})$ denote the corresponding Wiener space. Let $\mathbf{X}=\left(X_{0}, X_{1}, \cdots, X_{d}\right)$, where $\left\{X_{i}\right\}_{i \leqslant d+1}$ are bounded vector fields on a smooth finite dimensional Riemannian manifold $(\mathrm{N},\langle\cdot, \cdot\rangle)$. The pair $\left(\mathbf{X},\left\{B_{t}\right\}_{t \in \mathbb{R}_{+}}\right)$consists of a stochastic dynamical system (SDS) 
on $\mathrm{N}$ and it is $C^{j}(j \geqslant 1$ or $j=\infty)$ if all $X_{i}$ are $C^{j}$ bounded ([Elw82]). An N-valued semimartingale $\left\{x_{t}\right\}_{t \in \mathbb{R}_{+}}$defined up to a stopping time $\mathbf{e}^{x_{0}}$ is said to be a solution of the following Stratonovich SDE

$$
d x_{t}(\omega)=X_{0}\left(x_{t}(\omega)\right) d t+\sum_{i=1}^{d} X_{i}\left(x_{t}(\omega)\right) \circ d B_{t}^{i}(\omega)
$$

if for all $f \in C^{\infty}(\mathrm{N})$,

$$
f\left(x_{t}(\omega)\right)=f\left(x_{0}(\omega)\right)+\int_{0}^{t} X_{0} f\left(x_{s}(\omega)\right) d s+\int_{0}^{t} \sum_{i=1}^{d} X_{i} f\left(x_{s}(\omega)\right) \circ d B_{s}^{i}(\omega), \forall 0 \leqslant t<\mathbf{e}^{x_{0}}(w) .
$$

The solution to (2.2) always exists and is essentially unique when all $X_{i}$ 's are $C^{1}$ bounded $([$ Elw82] $)$. Moreover, for $\mathbb{P}$ almost all $\omega$, the mapping

$$
F_{t}(\cdot, \omega): x_{0}(\omega) \mapsto x_{t}(\omega)
$$

has the following property.

Proposition 2.3. ([Elw82, Chapter VIII]) Let $\left(\mathbf{X},\left\{B_{t}\right\}_{t \in \mathbb{R}_{+}}\right)$be a $C^{j} S D S$ on $\mathbf{N}$, where $j \geqslant 1$ or $j=\infty$. There is a version of the explosion time map $x \mapsto \mathbf{e}^{x}$, defined for $x \in \mathrm{N}$, and a version of $\left\{F_{t}(x, \omega)\right\}$, defined when $t \in\left[0, \mathbf{e}^{x}(\omega)\right)$, such that if $\mathrm{N}(t, \omega)=\{x \in \mathrm{N}: t<$ $\left.\mathbf{e}^{x}(w)\right\}$, then the following are true for each $(t, \omega) \in \mathbb{R}_{+} \times \Omega$.

i) The set $\mathrm{N}(t, \omega)$ is open in $\mathrm{N}$.

ii) For almost all $w, x_{0} \in \mathrm{N}$ and $0 \leqslant t<t^{\prime}<\mathbf{e}^{x_{0}}(w)$, we have the cocycle equality

$$
F_{t^{\prime}}\left(x_{0}, \omega\right)=F_{t^{\prime}-t}\left(x_{t}, \sigma_{t}(\omega)\right) \circ F_{t}\left(x_{0}, \omega\right),
$$

where $\sigma_{t}$ is the shift transformation on $\Omega$ :

$$
\sigma_{t}\left(\left(B_{s}^{1}, \cdots, B_{s}^{m}\right)_{s \geqslant 0}\right)=\left(\left(B_{t+s}^{1}, \cdots, B_{t+s}^{m}\right)_{s \geqslant 0}\right)-\left(B_{t}^{1}, \cdots, B_{t}^{m}\right) .
$$

iii) The map $F_{t}(x, \omega): \mathrm{N}(t, \omega) \rightarrow \mathrm{N}$ is $C^{j-1}$ (or $C^{\infty}$ when $j=\infty$ ) and is a diffeomorphism onto an open subset of $\mathrm{N}$. Moreover, the map $\tau \mapsto F_{\tau}(\cdot, \omega)$ of $[0, t]$ into $C^{j-1}$ (or $C^{\infty}$ when $j=\infty$ ) mappings of $\mathrm{N}(t, \omega)$ is continuous.

iv) For $1 \leqslant l \leqslant j-1$, denote by $D^{(l)} F_{t}(\cdot, \omega)$ the $l$-th tangent map of $F_{t}$. Then, for any $q \in[1, \infty)$, there is a bounded function $c_{l}(t, q)$, which depends on $t, m, q$, and the bounds of $\left\{\nabla^{\iota} X_{0}\right\}_{\iota \leqslant l},\left\{\nabla^{\iota} X_{i}\right\}_{1 \leqslant i \leqslant d, \iota \leqslant l+1}$ and $\left\{\nabla^{\iota-1} R\right\}_{1 \leqslant i \leqslant d, \iota \leqslant l+1}$ such that $\left\|\left[D^{(l)} F_{t}(\cdot, \omega)\right]\right\|_{L^{q}}<c_{l}(t, q)$, where $\|\cdot\|_{L^{q}}$ is the $L^{q}$-norm and $\nabla^{\iota}$ denotes the $\iota$-th covariant derivative and $R$ is the curvature tensor.

When $\mathrm{N}(t, \omega) \equiv \mathrm{N}$, the solution process $\left\{x_{t}\right\}$ to $(2.2)$ is said to be non-explosive. In this case, the maps $\left\{F_{t}(\cdot, \omega)\right\}_{t \in \mathbb{R}_{+}}$induce a kind of semi-flow on $\mathrm{N}$, which we shall call the stochastic flow associated to the $\operatorname{SDS}\left(\mathbf{X},\left\{B_{t}\right\}_{t \in \mathbb{R}_{+}}\right)$or (2.2). A direct consequence of Proposition 2.3 is the following regularity of a one-parameter family of stochastic flows. 
Corollary 2.4. Let $\left(\mathbf{X}^{a},\left\{B_{t}\right\}_{t \in \mathbb{R}_{+}}\right)$be a one-parameter family of $S D S$ on $\mathrm{N}$ with $\mathrm{N}^{a}(t, \omega) \equiv$ $\mathrm{N}$. Assume $X_{i}^{a}$ 's are all $C^{k}(k \geqslant 1$ or $=\infty)$ on $\mathrm{N} \times \mathrm{A}$ in the product differentiable structure. Then for any $t>0$, and $j \leqslant k-1, a \mapsto F_{t}^{a}(\cdot, \omega)$ is $C^{j}$ in the space of $C^{k-1-j}$ maps of $\mathrm{N}$.

Proof. Let $x_{t}^{a}$ be the solution for the $\operatorname{SDS}\left(\mathbf{X}^{a},\left\{B_{t}\right\}_{t \in \mathbb{R}_{+}}\right)$. Then $\left(x_{t}^{a}, a\right)$ solves the new $\operatorname{SDS}\left(\left(\mathbf{X}^{a}, 0\right),\left\{B_{t}\right\}_{t \in \mathbb{R}_{+}}\right)$on $\mathrm{N} \times \mathrm{A}$. The regularity in $a$ is a straightforward application of Proposition 2.3 by treating $a$ as a part of the initial value.

Corollary 2.4 does not apply when we only have Hölder continuity of $\mathbf{X}^{a}$ in $a$. However, it is still possible to discuss the regularities of $a \mapsto F_{t}^{a}(\cdot, \omega)$ by using one criterion from Kolmogorov:

Proposition 2.5. (cf. [Kun90, Theorem 1.4.1]) Let $T>0$ and let $\left\{\mathcal{Y}_{t}^{a}(\omega)\right\}_{t \in[0, T], a \in \mathrm{A}}$ be a one parameter family of random processes on a complete metric space, where A is some bounded n-dimensional Euclidean domain. Suppose there are positive constants $b, b_{0}, b_{1}, \cdots, b_{n}$, with $\sum_{i=0}^{n}\left(b_{i}\right)^{-1}<1$, and $\mathrm{C}_{0}(b)$ such that for all $t, t^{\prime} \in[0, T]$ and $a=$ $\left(a_{1}, \cdots, a_{n}\right), a^{\prime}=\left(a_{1}^{\prime}, \cdots, a_{n}^{\prime}\right) \in \mathrm{A}$,

$$
\mathbb{E}\left[\left|\mathcal{Y}_{t}^{a}-\mathcal{Y}_{t^{\prime}}^{a^{\prime}}\right|^{b}\right] \leqslant \mathrm{C}_{0}(b)\left(\left|t-t^{\prime}\right|^{b_{0}}+\sum_{i=1}^{n}\left|a_{i}-a_{i}^{\prime}\right|^{b_{i}}\right),
$$

then $\mathcal{Y}_{t}^{a}$ has a continuous modification with respect to the parameter $(t, a)$.

Let $\beta_{i}, i=0, \cdots, n$, be arbitrary positive numbers less than $b_{i}\left(1-\sum_{0}^{n}\left(b_{i}\right)^{-1}\right) / b$. Then for any hypercube $\mathrm{D}$ in $\mathrm{A}$, there exists a positive random variable $\mathrm{k}(\omega)$ with $\mathbb{E}\left[\mathrm{k}(\omega)^{b}\right]<\infty$ such that for any $t, t^{\prime} \in[0, T]$ and $a, a^{\prime} \in \mathrm{D}$,

$$
\left|\mathcal{Y}_{t}^{a}-\mathcal{Y}_{t^{\prime}}^{a^{\prime}}\right| \leqslant \mathrm{k}(\omega)\left(\left|t-t^{\prime}\right|^{\beta_{0}}+\sum_{i=1}^{n}\left|a_{i}-a_{i}^{\prime}\right|^{\beta_{i}}\right) .
$$

Next, we consider $\rho\left\langle 0, \varepsilon:=1 / \sqrt{-\rho}\right.$ and $\mathcal{L}_{\varepsilon}^{\prime}:=-\bar{X}+\varepsilon^{2} \Delta^{s}$. Extend $\mathcal{L}_{\varepsilon}^{\prime}$ to be a $G$ equivariant operator on $S \widetilde{M}$, which we shall denote by the same symbol. Its associated leafwise diffusions can be visualized using the classical Eells-Elworthy-Malliavin construction.

Recall that, for $\mathbf{v}=(x, \xi) \in S \widetilde{M}$, we have identified the stable manifold $\widetilde{W}^{s}(\mathbf{v})$ with $\widetilde{M} \times\{\xi\}$ and endowed it with the Riemannian metric on $\widetilde{M}$. In the same way, we can identify an orthogonal frame in the tangent space $T_{\mathbf{v}} \widetilde{W}^{s}$ with $O_{x} \times\{\xi\}$, where $O_{x}=\left(e_{1}, \cdots, e_{m}\right)$ is an element in $\mathcal{O}_{x}(\widetilde{M})$, the collection of the orthogonal frames in $T_{x} \widetilde{M}$. Set $\mathcal{O}^{s}(S \widetilde{M})$ for the bundle of such stable orthogonal frames:

$$
\mathcal{O}^{s}(S \widetilde{M}):=\left\{(x, \xi) \mapsto O_{x} \times\{\xi\}: O_{x}=\left(e_{1}, \cdots, e_{m}\right) \in \mathcal{O}_{x}(\widetilde{M}), x \in \widetilde{M}\right\} .
$$


We carry to $\mathcal{O}^{s}(S \widetilde{M})$ all the Riemannian geometry from $\mathcal{O}(\widetilde{M})=\bigcup_{x \in \widetilde{M}} \mathcal{O}_{x}(\widetilde{M})$. In particular, if $H_{x}$ denotes the horizontal lift from $T_{x} \widetilde{M}$ to $T_{O_{x}} \mathcal{O}(\widetilde{M})$, we can define the horizontal lift $\hat{H}_{\mathbf{v}}$ from $T_{\mathbf{v}} W^{s}$ to $T_{O_{x}, \xi} \mathcal{O}^{s}(S \widetilde{M})$ by $\hat{H}_{\mathbf{v}}(w, \xi)=\left(H_{x}(w), \xi\right)$ for $w \in T_{x} \widetilde{M}$.

Let $\left\{\left(B_{t}^{1}, \cdots, B_{t}^{m}\right)\right\}_{t \in \mathbb{R}_{+}}$be an $m$-dimensional Euclidean Brownian motion starting from the origin with the Euclidean Laplacian generator (and covariance matrix $2 t \mathrm{Id}$ ) and let $(\Omega, \mathbb{P})$ be the Wiener space. Set $\hat{X}$ as the horizontal lift of $\bar{X}$ to $T \mathcal{O}^{s}(S \widetilde{M})$. We can realize the diffusion for $\mathcal{L}_{\varepsilon}^{\prime}$ as the projection to $S \widetilde{M}$ of the non-explosive solution process $\left\{\mathrm{u}_{t}\right\}_{t \in \mathbb{R}_{+}}$ (the non-explosiveness follows since $(\widetilde{M}, \widetilde{g})$ has Ricci curvature uniformly bounded from below) to the Stratonovich $\mathrm{SDE}$ on $\mathcal{O}^{s}(S \widetilde{M})$ :

$$
d u_{t}=-\hat{X}\left(u_{t}\right) d t+\varepsilon \sum_{i=1}^{m} \hat{H}\left(u_{t}\left(e_{i}\right)\right) \circ d B_{t}^{i} .
$$

Let $\widehat{\pi}: \mathcal{O}^{s}(S \widetilde{M}) \rightarrow S \widetilde{M}$ be the natural projection and denote $\widehat{\mathcal{W}}^{s}$ for the foliation of $\mathcal{O}^{s}(S \widetilde{M})$ that projects on $\widetilde{\mathcal{W}}^{s}$. Let $D^{\infty}\left(\mathcal{O}^{s} S \widetilde{M}\right)$ be the space of homeomorphisms of $\mathcal{O}^{s}(S \widetilde{M})$ that preserve the leaves of $\widehat{\mathcal{W}}^{s}$ and are $C^{\infty}$-diffeomorphisms along the leaves. We endow $D^{\infty}\left(\mathcal{O}^{s} S \widetilde{M}\right)$ with the $C^{0, \infty}$ topology: $\varphi, \varphi^{\prime} \in D^{\infty}\left(\mathcal{O}^{s} S \widetilde{M}\right)$ are close if, for all $r>0$, the $r$-germs of $\varphi$ and $\varphi^{\prime}$ are uniformly close on compact sets and the $r$-germs of $\varphi^{-1}$ and $\left(\varphi^{\prime}\right)^{-1}$ are uniformly close on compact sets.

Proposition 2.6. With the above notations, for $\mathbb{P}$-a.e. $\omega \in \Omega$, for all $\varepsilon>0, t \geqslant 0$, there exists $\varphi_{\varepsilon, t}(\omega) \in D^{\infty}\left(\mathcal{O}^{s} S \widetilde{M}\right)$ such that the following hold true.

i) For all $u \in \mathcal{O}^{s}(S \widetilde{M}),(\omega, t) \mapsto \varphi_{\varepsilon, t}(\omega)(u)$ is a solution of the equation (2.3); in particular, for all $T \geqslant 0, \omega \mapsto \varphi_{\varepsilon, T}(\omega)$ is measurable with respect to the $\sigma$-algebra generated by $\left(B_{t}^{1}, \cdots, B_{t}^{m}\right), 0 \leqslant t \leqslant T$.

ii) For almost all $\omega$, all $t, s \geqslant 0, \varphi_{\varepsilon, t+s}(\omega)=\varphi_{\varepsilon, t}\left(\sigma_{s}(\omega)\right) \circ \varphi_{\varepsilon, s}(\omega)$.

iii) For all $\psi \in G, D \psi \circ \varphi_{\varepsilon, t}(\omega)=\varphi_{\varepsilon, t}(\omega) \circ D \psi$.

iv) The map $\varepsilon \mapsto \varphi_{\varepsilon, t}(\omega)$ is continuous in $D^{\infty}\left(\mathcal{O}^{s} S \widetilde{M}\right)$.

v) For fixed $r \in \mathbb{N}, t \geqslant 0$,

$$
\mathbb{E}\left[\max _{u}\left\|\left.\varphi_{\varepsilon, t}(\omega)(u)\right|_{\widehat{W}^{s}(u)}\right\|_{C^{r}}\right]<+\infty .
$$

Proof. Since both $\hat{X}$ and $\hat{H}$ are tangent to $\widehat{\mathcal{W}}^{s}$, the solution to $(2.3)$ is constrained in $\widehat{\mathcal{W}}^{s}$. For fixed $\xi$ and $\varepsilon$, equation $(2.3)$ can be seen as a $\operatorname{SDE}$ on $\mathcal{O}(\widetilde{M}) \times\{\xi\}$ and is solvable with infinite explosion time. Hence properties i) and ii) are given by Proposition 2.3. Property iii) follows from the uniqueness of the solution to (2.3). Considering $\varepsilon$ as a parameter, we get the continuity of the solution in $\varepsilon$ by Corollary 2.4. Considering $\xi$ as a parameter, the leaves of $\widehat{\mathcal{W}}^{s}$ and $\hat{X}, \widehat{H}$ vary Hölder continuously with respect to $\xi$. Hence, by a standard estimation using Burkholder inequality and Gronwall lemma and applying Proposition 2.5, we can obtain the continuity of the solution to $(2.3)$ in $\xi$, so that we can consider it as 
an element of $D^{\infty}\left(\mathcal{O}^{s} S \widetilde{M}\right)$. This shows iv). Finally we show v). Using a fundamental domain for the action of $G$ on $\widetilde{M}$, we may regard $\mathcal{O}^{s}(S M)$ as a subset of $\mathcal{O}^{s}(S \widetilde{M})$. By the $G$-equivariance property of the diffusion, we can restrict $u$ in the left hand side of $(2.4)$ to $\mathcal{O}^{s}(S M)$. By continuity of $\left.\varphi_{\varepsilon, t}(\omega)(u)\right|_{\widehat{W}^{s}(u)}$ in $u$, the compactness of $\mathcal{O}^{s}(S M)$ and Proposition 2.5, for (2.4), it suffices to show for each $u \in \mathcal{O}^{s}(S M), r \in \mathbb{N}$ and $t>0$,

$$
\mathbb{E}\left[\left\|\left.\varphi_{\varepsilon, t}(\omega)(u)\right|_{\widehat{W}^{s}(u)}\right\|_{C^{r}}\right]<+\infty .
$$

This is an application of Proposition 2.3 iv) by using the SDE (2.3).

Equation (2.3) for $\varepsilon=0$ is the ordinary differential equation $d u_{t}=-\hat{X}\left(u_{t}\right) d t$. Its solution is the extension $\left\{\widehat{\boldsymbol{\Phi}}_{-t}\right\}_{t \in \mathbb{R}}$ of the reversed geodesic flow to $\mathcal{O}^{s}(S \widetilde{M})$ by parallel transportation along the geodesics, and is called the reversed stable frame flow. Write $\nu_{\rho}$ for the probability measure on $D^{\infty}\left(\mathcal{O}^{s} S \widetilde{M}\right)$ that is the distribution of $\varphi_{\varepsilon, 1}=\varphi_{1 / \sqrt{-\rho}, 1}$ in Proposition 2.6. Every element $\varphi \in D^{\infty}\left(\mathcal{O}^{s} S \widetilde{M}\right)$ preserves each leaf $\widehat{W}^{s}(u)$ and is a $C^{\infty}$ diffeomorphism along it. We write $J(\varphi, u)$ for the Jacobian determinant of the tangent map of $\left.\varphi\right|_{\widehat{W}^{s}(u)}$ at $u$. For later use, we state a proposition concerning the limit behavior of $\varphi_{1 / \sqrt{-\rho}, t}$ when $\rho \rightarrow-\infty$.

Proposition 2.7. With the above notations, the following are true.

i) For $\mathbb{P}$-a.e. $\omega \in \Omega$, all $t>0$, as $\rho \rightarrow-\infty, \varphi_{1 / \sqrt{-\rho}, t}(\omega)$ converge to $\hat{\mathbf{\Phi}}_{-t}$ in $D^{\infty}\left(\mathcal{O}^{s} S \widetilde{M}\right)$, in particular, $\varphi_{1 / \sqrt{-\rho}, 1}$ converge to the time 1 map of the reversed stable frame flow.

ii) For any $-\infty<\rho<0$ and $r, \mathrm{M}$ positive integers,

$B_{r, \mathrm{M}}(\rho):=\mathbb{E}\left[\max _{u}\left\|\left.\varphi_{1 / \sqrt{-\rho}, \mathrm{M}}(\omega)(u)\right|_{\widehat{W}^{s}(u)}\right\|_{C^{r}}\right]<+\infty$ and $B_{r, \mathrm{M}}:=\limsup _{\rho \rightarrow-\infty} B_{r, \mathrm{M}}(\rho)<+\infty$.

iii) For any $r \in \mathbb{N}$,

$$
\lim _{\rho \rightarrow-\infty} \int \max _{u}\left|\left\|\left.\varphi\right|_{\widehat{W}^{s}(u)}\right\|_{C^{r}}-\left\|\left.\widehat{\mathbf{\Phi}}_{-1}\right|_{\widehat{W}^{s}(u)}\right\|_{C^{r}}\right| d \nu_{\rho}(\varphi)=0 .
$$

iv) We have

$$
\lim _{\rho \rightarrow-\infty} \int \log J(\varphi, u) d \nu_{\rho}(\varphi)=\log J\left(\hat{\mathbf{\Phi}}_{-1}, u\right)
$$

and the convergence is locally uniform in $u$.

Proof. The proof of continuity of the solution to (2.3) in Proposition 2.6 extends to $\varepsilon=0$. This shows i). When $\rho \rightarrow-\infty, \varepsilon=1 / \sqrt{-\rho} \rightarrow 0$. For ii), note that $B_{r, \mathrm{M}}(\rho)$ is finite by Proposition $2.6 \mathrm{v}$ ) (applied for $t=\mathrm{M}$ ). Then $B_{r, \mathrm{M}}$ is also finite by using the continuity in $(\varepsilon, u)$ in the estimation of the expectation in (2.5) in the proof of Proposition $2.6 \mathrm{v}$ ). Similarly, we have the continuity in $\varepsilon$ of the tangent maps (and their derivatives in $\varepsilon$ ) of the solution to (2.3). Following Proposition 2.3 iv), it is easy to deduce from (2.3) the 
continuity in $\varepsilon$ of the norm of the tangent maps and of the Jacobian of the first order tangent map. This shows iii) and iv).

It follows from Proposition 2.6 i) and ii) that we can consider $\varphi_{\varepsilon, n}, n \in \mathbb{N}$, as an independent product of the homeomorphisms $\varphi_{\varepsilon, 1}$ and that we can apply the theory of independent random mappings. Let $\hat{\pi}$ be the projection map from $\mathcal{O}^{s}(S \widetilde{M})$ to $S \widetilde{M}$. For any $C^{2}$ compactly supported function $f$ on $S \widetilde{M},(x, \xi) \in S \widetilde{M}$ and any frame $u \in \mathcal{O}^{s}(S \widetilde{M})$ in the fiber $\hat{\pi}^{-1}(x, \xi)$, we have

$$
\int_{S \widetilde{M}} f(y, \eta) d \mathbf{p}_{\rho}(\varepsilon,(x, \xi), d(y, \eta))=\int_{D^{\infty}\left(\mathcal{O}^{s} S \widetilde{M}\right)} f(\bar{\pi} \varphi(u)) d \nu_{\rho}(\varphi) .
$$

Let $\widehat{\mathbf{m}}_{\rho}$ be the measure on $\mathcal{O}^{s}(S \widetilde{M})$ that projects on $\widetilde{\mathbf{m}}_{\rho}$ on $S \widetilde{M}$ and such that the conditional measures on fibers of the projection map $\hat{\pi}$ are proportional to the Lebesgue measure on $m$-dimensional frames. The following is true.

Proposition 2.8. The measure $\widehat{\mathbf{m}}_{\rho}$ is stationary under $\nu_{\rho}$, i.e., it satisfies, for any $C^{2}$ compactly supported function $f$ on $\mathcal{O}^{s}(S \widetilde{M})$,

$$
\int f(\varphi(u)) d \nu_{\rho}(\varphi) d \widehat{\mathbf{m}}_{\rho}(u)=\int f(u) d \widehat{\mathbf{m}}_{\rho}(u) .
$$

Moreover, the conditional measures $\widehat{\mathbf{m}}_{\rho, u}^{s}$ of $\widehat{\mathbf{m}}_{\rho}$ with respect to the leaves of the $\widehat{\mathcal{W}}^{s}$ foliation are absolutely continuous with respect to Lebesgue.

Proof. The stationarity follows from relation (2.7), the stationarity of $\widetilde{\mathbf{m}}_{\rho}$ and the fact that the flow preserves the orthogonal group on the fibers ([CE86, Lemma 3.1]). The leaves of $\widehat{\mathcal{W}}^{s}$ are made of whole fibers and project on the leaves of $\widetilde{\mathcal{W}}^{s}$. The conditional measures on the leaves of $\widehat{\mathcal{W}}^{s}$ are given by the extension by Lebesgue on the fibers of the conditional measures on the leaves of $\widetilde{\mathcal{W}}^{s}$. By Proposition 2.1 iii), they are therefore absolutely continuous.

Let $\pi: \mathcal{O}^{s}(S M) \rightarrow S M$ be the quotient of the map $\hat{\pi}$ by the action of $G$ and let $\overline{\mathcal{W}}^{s}=$ $\left\{\bar{W}^{s}(u)\right\}_{u \in O^{s}(S M)}$ denote the corresponding quotient foliation of $\widehat{\mathcal{W}}^{s}$. Let $D^{\infty}\left(\mathcal{O}^{s} S M\right)$ be the space of homeomorphisms of $\mathcal{O}^{s}(S M)$ that preserve the leaves of $\overline{\mathcal{W}}^{s}$ and are $C^{\infty}$ diffeomorphisms along the leaves. We endow $D^{\infty}\left(\mathcal{O}^{s} S M\right)$ with the $C^{0, \infty}$ topology: $\varphi, \varphi^{\prime} \in$ $D^{\infty}\left(\mathcal{O}^{s} S M\right)$ are close if, for all $r>0$, the $r$-germs of $\varphi$ and $\varphi^{\prime}$ are uniformly close and the $r$-germs of $\varphi^{-1}$ and $\left(\varphi^{\prime}\right)^{-1}$ are uniformly close. By Proposition 2.6 iii), we can consider $\nu_{\rho}$ as a probability measure on $D^{\infty}\left(\mathcal{O}^{s} S M\right)$.

We define the measure $\overline{\mathbf{m}}_{\rho}$ on $\mathcal{O}^{s}(S M)$ such that its $G$-invariant extension to $\mathcal{O}^{s}(S \widetilde{M})$ is $\widehat{\mathbf{m}}_{\rho}$. We see that $\overline{\mathbf{m}}_{\rho}$ is a probability measure that projects to $\mathbf{m}_{\rho}$ on $S M$ and is such that the conditional measures on fibers of $\pi$ are proportional to Lebesgue on $m$-dimensional frames. As a consequence of Proposition 2.8, we have 
Corollary 2.9. The measure $\overline{\mathbf{m}}_{\rho}$ is stationary under $\nu_{\rho}$, i.e., it satisfies, for any continuous function $f$ on $\mathcal{O}^{s}(S M)$,

$$
\int f(\varphi u) d \nu_{\rho}(\varphi) d \overline{\mathbf{m}}_{\rho}(u)=\int f(u) d \overline{\mathbf{m}}_{\rho}(u) .
$$

Moreover, the conditional measures $\overline{\mathbf{m}}_{\rho, u}^{s}$ of $\overline{\mathbf{m}}_{\rho}$ with respect to the leaves of the $\overline{\mathcal{W}}^{s}$ foliation are absolutely continuous with respect to Lebesgue.

We are interested in the limit measures of $\mathbf{m}_{\rho}$ 's when $\rho$ goes to $-\infty$. Let $\mathbf{m}$ be such a limit point and let $\overline{\mathbf{m}}$ be the probability measure on $\mathcal{O}^{s}(S M)$ that projects to $\mathbf{m}$ on $S M$ and is such that the conditional measures on fibers of $\pi$ are proportional to the Lebesgue measure on $m$-dimensional frames. Then $\overline{\mathbf{m}}$ is the limit of $\overline{\mathbf{m}}_{\rho}$ along the same subsequence. Let $\left\{\overline{\boldsymbol{\Phi}}_{-t}\right\}_{t \in \mathbb{R}}$ be the reversed stable frame flow. Then $\overline{\mathbf{m}}$ is invariant under $\overline{\mathbf{\Phi}}_{-t}$. To show $\mathbf{m}$ is Liouville, it suffices to show the conditional measures of $\overline{\mathbf{m}}$ on the leaves of $\overline{\mathcal{W}}^{s}$ are absolutely continuous with respect to Lebesgue. But this does not follow from Corollary 2.9 by the same reason that we mentioned in Remark 2.2 . What we are going to do in the next section is to analyze the entropy related to the natural random dynamics for $\overline{\mathbf{m}}_{\rho}$ that arises in the stationarity relation (2.8).

\section{ENTROPY OF RANDOM MAPPINGS}

We consider the action on $\mathcal{O}^{s}(S M)$ of the random elements of $D^{\infty}\left(\mathcal{O}^{s} S M\right)$ with distribution $\nu_{\rho},-\infty \leqslant \rho<0$. Namely, let $\mathcal{S}:=\left(D^{\infty}\left(\mathcal{O}^{s} S M\right)\right)^{\mathbb{N} \cup\{0\}}$, endowed with the product measures $\nu_{\rho}^{\otimes \mathbb{N} \cup\{0\}}$ (with the convention that $\nu_{-\infty}$ is the Dirac measure at $\overline{\boldsymbol{\Phi}}_{-1}$ ) and the shift transformation $\sigma$. On the space $\mathcal{T}:=\mathcal{S} \times \mathcal{O}^{s}(S M)$, define the transformation $\tau$ by:

$$
\tau(\underline{\varphi}, u):=\left(\sigma \underline{\varphi}, \varphi_{0} u\right) \text {. }
$$

For $-\infty<\rho<0$, let $\overline{\mathbf{m}}_{\rho}$ be the stationary measure from Corollary 2.9 and for $\rho=-\infty$, let $\overline{\mathbf{m}}_{-\infty}=\overline{\mathbf{m}}$ be some weak* limit of $\overline{\mathbf{m}}_{\rho}$ as $\rho \rightarrow-\infty$. For $-\infty \leqslant \rho<0$, the measure

$\mu_{\rho}:=\nu_{\rho}^{\otimes \mathbb{N} \cup\{0\}} \otimes \overline{\mathbf{m}}_{\rho}$ is invariant under the transformation $\tau$.

Let $\mathcal{P}$ be a measurable partition of $\mathcal{T}$ with finite or countably many elements. We assume $-\int \log \left(\overline{\mathbf{m}}_{\rho}(\mathcal{P})\right) d \mu_{\rho}<+\infty$. For $n \in \mathbb{N}$, set $\mathcal{P}_{-1}=\mathcal{P}$ and $\mathcal{P}_{-n}:=\mathcal{P} \bigvee \tau^{-1} \mathcal{P} \bigvee \cdots \bigvee \tau^{-(n-1)} \mathcal{P}$ for $n>1$, where $\bigvee$ denotes the join of partitions, i.e., the refinement of partitions by taking intersections. For $(\underline{\varphi}, u) \in \mathcal{T}$, let $\mathcal{P}_{-n}(\underline{\varphi}, u)$ denote the element of $\mathcal{P}_{-n}$ that contains $(\underline{\varphi}, u)$. We define the entropy $h_{\rho}^{s}$ for $\overline{\mathbf{m}}_{\rho}$ as

$$
h_{\rho}^{s}:=\sup _{\mathcal{P}} \underline{h}_{\rho, \mathcal{P}}^{s},
$$

where

$$
\underline{h}_{\rho, \mathcal{P}}^{s}:=\liminf _{n \rightarrow+\infty}-\frac{1}{n} \int \log \overline{\mathbf{m}}_{\rho, u}^{s}\left(\mathcal{P}_{-n}(\underline{\varphi}, u)\right) d \mu_{\rho}(\underline{\varphi}, u) .
$$


For a formal definition of $\overline{\mathbf{m}}_{\rho, u}^{s}$, we should use a measurable partition $\mathcal{R}$ subordinated to $\overline{\mathcal{W}}^{s}$ (see Section 4 for details). But the value of $\underline{h}_{\rho, \mathcal{P}}^{s}$ does not depend on the choice of such a subordinated partition and is thus well-defined. Observe that

$$
-\int \log \overline{\mathbf{m}}_{\rho, u}^{s}\left(\mathcal{P}_{-n}(\underline{\varphi}, u)\right) d \mu_{\rho}(\underline{\varphi}, u) \leqslant-\int \log \overline{\mathbf{m}}_{\rho}\left(\mathcal{P}_{-n}(\underline{\varphi}, u)\right) d \mu_{\rho}(\underline{\varphi}, u) .
$$

Using the random Ruelle inequality (cf. [BB95, Kif86]), we obtain that $\underline{h}_{\rho, \mathcal{P}}^{s}$ is bounded independent of $\mathcal{P}$. Hence $h_{\rho}^{s}$ is finite. Note also that $\overline{\mathbf{m}}_{\rho, u}^{s}$ is absolutely continuous with respect to Lebesgue with a smooth density.

For the computation of $\underline{h}_{\rho, \mathcal{P}}^{s}$, we can restrict the conditional measure $\overline{\mathbf{m}}_{\rho, u}^{s}$ to the local stable leaf $\bar{W}_{\text {loc, } \epsilon}^{s}(u):=\left\{w \in \bar{W}^{s}(u): d_{\bar{W}^{s}}(w, u)<\epsilon\right\}$ for $\epsilon$ small enough. Recall that $\varphi \in D^{\infty}\left(\mathcal{O}^{s} S M\right)$ preserves each leaf $\bar{W}^{s}(u)$ and is a $C^{\infty}$ diffeomorphism along it. Write $J(\varphi, u)$ for the Jacobian determinant of the tangent map of $\left.\varphi\right|_{W^{s}(u)}$ at $u$. We will conclude Theorem 1.1 from the following two propositions.

Proposition 3.1. For $-\infty<\rho<0$,

$$
h_{\rho}^{s} \geqslant \int \log J(\varphi, u) d \nu_{\rho}(\varphi) d \overline{\mathbf{m}}_{\rho}(u) .
$$

Proposition 3.2. Let $\rho_{p}, p \in \mathbb{N}$, be a sequence such that $\rho_{p} \rightarrow-\infty$ and $\mathbf{m}_{\rho_{p}}$ converge to the probability measure $\mathbf{m}$ as $p \rightarrow+\infty$, and let $\overline{\mathbf{m}}$ be as above. Then

$$
h_{\overline{\mathbf{m}}}^{s}:=h_{-\infty}^{s} \geqslant \limsup _{p \rightarrow+\infty} h_{\rho_{p}}^{s} .
$$

The proofs of Proposition 3.1 and Proposition 3.2 use completely different techniques and will be presented in this section and the following section, respectively.

In the following, we shall use $H_{\vartheta}(\mathcal{A})$ to denote the entropy of a measurable partition $\mathcal{A}$ with respect to a measure $\vartheta$ of some space and use $H_{\vartheta}(\mathcal{A} \mid \mathcal{B})$ to denote the entropy of $\mathcal{A}$ conditioned on some measurable partition $\mathcal{B}$, whenever these entropies are well-defined. We shall denote $\bar{m}$ for the dimension of $\bar{W}^{s}$; for $(\underline{\varphi}, u) \in \mathcal{T}$, we shall write

$$
\left.\underline{\varphi}\right|_{0}=\mathrm{Id} \text { and }\left.\underline{\varphi}\right|_{n}=\varphi_{n-1} \circ \cdots \circ \varphi_{0}, \forall n \geqslant 1,
$$

and $J\left(\left.\underline{\varphi}\right|_{n}, u\right)$ for the Jacobian determinant of the tangent map of $\left.\left.\underline{\varphi}\right|_{n}\right|_{\bar{W}^{s}(u)}$ at $u$. Clearly, we have $J\left(\left.\underline{\varphi}\right|_{1}, u\right)=J\left(\varphi_{0}, u\right)$ for $\underline{\varphi}=\left(\varphi_{0}, \varphi_{1}, \cdots\right) \in \mathcal{S}$.

Proof of Theorem 1.1. Let $\rho_{p}, p \in \mathbb{N}$, be a sequence such that $\rho_{p} \rightarrow-\infty$ and $\mathbf{m}_{\rho_{p}}$ converge to the probability measure $\mathbf{m}$ as $p \rightarrow+\infty$, and let $\overline{\mathbf{m}}$ be as above. Recall that $\overline{\mathbf{\Phi}}_{-1}$ is the time one map of the reversed frame flow on $\mathcal{O}^{s}(S M)$ which is a compact isometric extension of the time one map of the reversed geodesic flow $\boldsymbol{\Phi}_{-1}$ on $S M$. Hence,

$$
h_{\mathbf{m}}=h_{\overline{\mathbf{m}}} \text {. }
$$


On the other hand, we have:

$$
h_{\overline{\mathbf{m}}}=\sup _{\mathcal{P}} \lim _{n \rightarrow+\infty} \frac{1}{n} H_{\overline{\mathbf{m}}}\left(\mathcal{P}_{-n}\right) \geqslant \sup _{\mathcal{P}} \liminf _{n \rightarrow+\infty}-\frac{1}{n} \int \log \overline{\mathbf{m}}_{u}^{s}\left(\mathcal{P}_{-n}(u)\right) d \overline{\mathbf{m}}(u)=h_{\overline{\mathbf{m}}}^{s} .
$$

Assume Proposition 3.1 and Proposition 3.2 hold true. Then,

$$
h_{\overline{\mathbf{m}}}^{s} \geqslant \limsup _{p \rightarrow+\infty} \int \log J(\varphi, u) d \nu_{\rho}(\varphi) d \overline{\mathbf{m}}_{\rho_{p}}(u)=\int \log J\left(\overline{\mathbf{\Phi}}_{-1}, u\right) d \overline{\mathbf{m}}(u),
$$

where the last equality holds by Proposition 2.7 iii). Altogether, we obtain

$$
h_{\mathbf{m}} \geqslant \int \log J\left(\overline{\mathbf{\Phi}}_{-1}, u\right) d \overline{\mathbf{m}}(u) .
$$

Note that $\overline{\mathcal{W}}^{s}$ is the central unstable foliation for $\overline{\boldsymbol{\Phi}}_{-1}$, so that $\int \log J\left(\overline{\boldsymbol{\Phi}}_{-1}, u\right) d \overline{\mathbf{m}}(u)$ is the integral of the sum of the nonnegative exponents of $\overline{\mathbf{\Phi}}_{-1}$ for $\overline{\mathbf{m}}$; neither the direction of the flow nor the vertical directions tangent to the fibers provide positive exponents, so that $\int \log J\left(\overline{\mathbf{\Phi}}_{-1}, u\right) d \overline{\mathbf{m}}(u)$ is the integral of the sum of the positive exponents of $\boldsymbol{\Phi}_{-1}$ for $\mathbf{m}$. By $[\mathbf{B R 7 5}], \mathbf{m}$ is the normalized Liouville measure.

3.1. Proof of Proposition 3.1. The deterministic diffeomorphism version estimation of (3.2) is standard using Pesin theory (cf. [Man83]). But this cannot be used directly since we are in the random and non-invertible case.

Clearly, Proposition 3.1 would follow if we can show the sample measures are SRB. This approach might work since in a similar context, Blumenthal-Young ([BY19]) showed the sample measures are SRB. We didn't try that way since we don't need that strong conclusion and the intuition for Proposition 3.1 is relatively simpler.

For a non-invertible endomorphism of a compact manifold preserving an absolutely continuous measure, the corresponding measure theoretical entropy is at least the integral of the logarithm of the Jacobian, which coincides with the so-called folding entropy (cf. [Rue96], [LS11]). Proposition 3.1 is intuitively a random conditional version of this phenomenon. But it might be subtle since we are considering the conditional measures and are in the random case. So we will give some details for the key steps.

We first recall some notations and results concerning Pesin local Lyapunov charts theory for random diffeomorphisms. In many places, we have to take invariant variables instead of constants since our system $\left(\mathcal{T}, \tau, \mu_{\rho}\right)$ is invariant, but not necessarily ergodic in general.

Lemma 3.3. ([Ose68]) For each $\rho<0$, there is a measurable $\boldsymbol{\Omega} \subset \mathcal{T}$ with $\mu_{\rho}(\boldsymbol{\Omega})=1$ such that for $(\underline{\varphi}, u) \in \mathbf{\Omega}$, there exist $r(\underline{\varphi}, u) \in \mathbb{N}$ and, for $i, 1 \leqslant i \leqslant r(\underline{\varphi}, u), \chi_{i}(\underline{\varphi}, u), d_{i}(\underline{\varphi}, u)$ and a filtration

$$
\{0\}=V_{r(\underline{\varphi}, u)+1} \subset V_{r(\underline{\varphi}, u)} \subset \cdots \subset V_{1}=T_{u} \bar{W}^{s}(u)
$$

with the following properties:

i) all of $r, \chi_{i}, d_{i}, V_{i}$ 's depend measurably on $(\varphi, u)$;

ii) $\lim _{n \rightarrow+\infty} \frac{1}{n} \log \left\|D_{u}\left(\left.\underline{\varphi}\right|_{n}\right)(e)\right\|=\chi_{i}(\underline{\varphi}, u)$ for $e \in V_{i}(\underline{\varphi}, u) \backslash V_{i+1}(\underline{\varphi}, u)$; 
iii) $d_{i}(\underline{\varphi}, u)=\operatorname{dim} V_{i}(\underline{\varphi}, u)-\operatorname{dim} V_{i+1}(\underline{\varphi}, u)$ and $\sum_{i=1}^{r(\underline{\varphi}, u)} d_{i}(\underline{\varphi}, u)=\bar{m}$;

iv) $\int \sum_{i=1}^{r(\underline{\varphi}, u)} \chi_{i}(\underline{\varphi}, u) d_{i}(\underline{\varphi}, u) d \mu_{\rho}(\underline{\varphi}, u)=\int \log J(\varphi, u) d \nu_{\rho}(\varphi) d \overline{\mathbf{m}}_{\rho}(u)$.

Lemma 3.4. (cf. [LQ95, Chapter III, Section 1]) For each $\rho<0$, given a small enough positive $\tau$-invariant function $\epsilon$ on $\mathcal{T}$, there is a positive function $\kappa$ on $\Omega \times\{\mathbb{N} \cup\{0\}\}$ such that for $n \in \mathbb{N} \cup\{0\}$,

$$
\kappa((\underline{\varphi}, u), n+1) \leqslant e^{\epsilon} \cdot \kappa((\underline{\varphi}, u), n),
$$

a positive constant $\kappa_{0}$ and a sequence Euclidean metrics $\|\cdot\|_{(\underline{\varphi}, u), n}^{\prime}$ on $T_{\left.\underline{\varphi}\right|_{n}(u)} \bar{W}^{s}\left(\left.\underline{\varphi}\right|_{n}(u)\right)$ such that for all $n \in \mathbb{N} \cup\{0\}$,

i) $\kappa_{0}\|\cdot\|_{\left.\underline{\varphi}\right|_{n}(u)} \leqslant\|\cdot\|_{(\varphi, u), n}^{\prime} \leqslant \kappa((\underline{\varphi}, u), n)\|\cdot\|_{\left.\underline{\varphi}\right|_{n}(u)}$, where $\|\cdot\|_{\left.\underline{\varphi}\right|_{n}(u)}$ is the Riemannian norm on $T_{\left.\underline{\varphi}\right|_{n}(u)} \bar{W}^{\bar{s}}\left(\left.\underline{\varphi}\right|_{n}(u)\right)$;

ii) $\mathbf{F}_{(\underline{\varphi}, u), n}(e):=\exp _{\left.\varphi\right|_{n+1}(u)}^{-1} \circ \varphi_{n} \circ \exp _{\left.\underline{\varphi}\right|_{n}(u)}(e)$ is defined for e with $\|e\|_{(\varphi, u), n}^{\prime} \leqslant \epsilon(\underline{\varphi}, u)$;

iii) $\mathbf{F}_{(\underline{\varphi}, u), n}$ is $C^{2}$ and $\left\|D^{(2)} \mathbf{F}_{(\underline{\varphi}, u), n}\right\|_{(\underline{\varphi}, u), n, n+1}^{\prime}<\kappa((\underline{\varphi}, u), n)$, where by $\|\cdot\|_{(\underline{\varphi}, u), n, n+1}^{\prime}$ we mean the norm of the tangent map calculated using $\|\cdot\|_{(\underline{\varphi}, u), n}^{\prime}$ and $\|\cdot\|_{(\underline{\varphi}, u), n+1}^{\prime}$;

iv) the map $D \mathbf{F}_{(\underline{\varphi}, u), n}$ satisfies

$$
\left\|D_{e} \mathbf{F}_{(\underline{\varphi}, u), n}-D_{0} \mathbf{F}_{(\underline{\varphi}, u), n}\right\|_{(\underline{\varphi}, u), n, n+1}^{\prime} \leqslant \epsilon(\underline{\varphi}, u)\|e\|_{(\underline{\varphi}, u), n}^{\prime}
$$

v) the map $D_{0} \mathbf{F}_{(\underline{\varphi}, u), n}$ satisfies

$$
e^{\chi_{i}(\underline{\varphi}, u)-\epsilon(\underline{\varphi}, u)}\|e\|_{(\underline{\varphi}, u), n}^{\prime} \leqslant\left\|D_{0} \mathbf{F}_{(\underline{\varphi}, u), n}(e)\right\|_{(\underline{\varphi}, u), n+1}^{\prime} \leqslant e^{\chi_{i}(\underline{\varphi}, u)+\epsilon(\underline{\varphi}, u)}\|e\|_{(\underline{\varphi}, u), n}^{\prime},
$$

for all $e \in E_{i}\left(\tau^{n}(\underline{\varphi}, u)\right)$. Moreover, for $i, 1 \leqslant i \leqslant r(\underline{\varphi}, u)$, the spaces $E_{j}(\underline{\varphi}, u), j \geqslant i$, generate $V_{i}(\underline{\varphi}, u)$.

(Since elements of $D^{\infty}\left(\mathcal{O}^{s} S M\right)$ preserve the leaves of $\overline{\mathcal{W}}^{s}$, Lemma 3.4 can be obtained as in [LQ95, Chapter III, Section 1]) using the natural auxiliary charts $E_{j}(\underline{\varphi}, u)^{\prime} s$ and Lemma 3.3.)

For Proposition 3.1, we shall follow Mañé ([Man83]) to give a local version of (3.2) for Bowen balls defined using the norms in Lemma 3.4 and then compare it with local entropy for special partitions. Note that we are in the non-invertible case, $\kappa$ is not invariant (i.e., for $(\varphi, u) \in \boldsymbol{\Omega}, \kappa((\underline{\varphi}, u), n)$ does not equal to $\kappa\left(\tau^{n}(\underline{\varphi}, u), 0\right)$ in general). To overcome this deficiency, we will pick up a set A with measure close to 1 and define modified Bowen balls associated to A.

Let $\epsilon_{0}>0,-\infty<\rho<0$ be fixed. Choose $\epsilon<\epsilon_{0}$ as in Lemma 3.4. For any $a \in(0,1)$, we choose a measurable set $\mathrm{A} \subset \boldsymbol{\Omega}$ with $\mu_{\rho}(\mathrm{A})>1-a$ as follows. By the ergodicity of $\nu_{\rho}^{\otimes \mathbb{N} \cup\{0\}}$ with respect to $\sigma$ and the integrability property (2.6), for $\nu_{\rho}^{\otimes \mathbb{N} \cup\{0\}}$ almost every $\underline{\varphi}$,

$$
\lim _{n \rightarrow+\infty} \frac{1}{n} \sum_{i=1}^{n}\left(\log \max _{u}\left\|\left.\varphi_{i}\right|_{\widehat{W}^{s}(u)}\right\|_{C^{2}}\right)^{+}=\int\left(\log \max _{u}\left\|\left.\varphi\right|_{\widehat{W}^{s}(u)}\right\|_{C^{2}}\right)^{+} d \nu_{\rho}(\varphi)=: L<+\infty .
$$


For any $b>0$, let

$$
\mathrm{A}(b):=\left\{\underline{\varphi} \in \mathcal{S}: \prod_{i=0}^{n-1} \max _{u}\left\|\left.\varphi_{i}\right|_{W^{s}(u)}\right\|_{C^{2}} \leqslant b e^{2 L n} \text { for all } n \in \mathbb{N} \cup\{0\}\right\} .
$$

Then there exists $b>0$ large such that

$$
\nu_{\rho}^{\otimes N} \cup\{0\}(\mathrm{A}(b))>1-\frac{1}{2} a .
$$

Let $b$ be as in (3.4). For $l>0$, set

$$
\mathrm{A}(b, l):=\left\{(\underline{\varphi}, u) \in \Omega: \underline{\varphi} \in \mathrm{A}(b), \eta \kappa_{0}(\kappa((\underline{\varphi}, u), 0))^{-2}>l\right\} .
$$

Choosing $\eta>0, \epsilon(\underline{\varphi}, u)$ with $\epsilon(\underline{\varphi}, u)<\epsilon_{0}$ and $l$ to be small enough, we can obtain a measurable set

$$
\mathrm{A}:=\mathrm{A}(b, l) \cap\{(\underline{\varphi}, u): \eta<\epsilon(\underline{\varphi}, u)\}
$$

with $\mu_{\rho}$ measure greater than $1-a$.

Let $\mathrm{A}$ be as in (3.6). For $\mu_{\rho}$ almost all $(\underline{\varphi}, u) \in \mathrm{A}$, it will return to A under the iterations of the map $\tau$ for infinitely many times. Hence, for any such $(\underline{\varphi}, u)$ and $k \geqslant 1$,

$$
\|\cdot\|_{(\underline{\varphi}, u), k}^{\prime \prime}:=\|\cdot\|_{\tau_{k}^{\mathrm{N}}(\underline{\varphi}, u), k-\mathrm{N}_{k}^{\mathrm{A}}}^{\prime}
$$

is well-defined, where $\mathrm{N}_{k}^{\mathrm{A}}$ is the last non-negative time before or equal to $k$ with $\tau^{\mathrm{N}_{k}^{\mathrm{A}}}(\underline{\varphi}, u) \in$ A. For $\eta>0,(\underline{\varphi}, u) \in \mathrm{A}$ such that $\eta<\epsilon(\underline{\varphi}, u)$, and $n \in \mathbb{N}$, let us define the modified random $\overline{\mathcal{W}}^{s}$-Bowen ball (with respect to $\mathrm{A}$ ) by

$$
\begin{aligned}
\mathbf{B}_{\mathrm{A}}^{s}(\underline{\varphi}, u, \eta, n):=\left\{e \in T_{u} \bar{W}^{s}(u):\right. & \|e\|_{(\underline{\varphi}, u), 0}^{\prime \prime}<\eta \kappa^{-1}((\underline{\varphi}, u), 0), \text { and for } k, 1 \leqslant k \leqslant n, \\
& \left.\left\|\left.\mathbf{F}_{(\underline{\varphi}, u)}\right|_{k}(e)\right\|_{(\underline{\varphi}, u), k}^{\prime \prime}<\eta \kappa^{-1}\left(\tau^{\mathrm{N}_{k}^{\mathrm{A}}}(\underline{\varphi}, u), k-\mathrm{N}_{k}^{\mathrm{A}}\right)\right\},
\end{aligned}
$$

where $\left.\mathbf{F}_{(\underline{\varphi}, u)}\right|_{k}:=\mathbf{F}_{(\underline{\varphi}, u), k-1} \circ \cdots \circ \mathbf{F}_{(\underline{\varphi}, u), 0}$.

The following can be considered as a first step coarse local version of Proposition 3.1:

Lemma 3.5. Let $-\infty<\rho<0, a \in(0,1), \epsilon_{0}>0$, and $\eta>0$ be fixed. Choose $\epsilon<\epsilon_{0}$ as in Lemma 3.4. Let $\mathrm{A} \subset \boldsymbol{\Omega}$ be as in (3.6). Then, there is a positive geometric constant $\mathbf{C}_{0}$ such that, setting $\mathbf{c}_{0}:=\frac{1}{2} \bar{m} \log \frac{l \kappa_{0}}{\eta}$, for $\mu_{\rho}$ almost all $(\underline{\varphi}, u)$ and all $n \in \mathbb{N}$,

$$
-\frac{1}{n} \log \overline{\mathbf{m}}_{\rho, u}^{s}\left(\exp _{u}\left(\mathbf{B}_{\mathrm{A}}^{s}(\underline{\varphi}, u, \eta, n)\right)\right) \geqslant-\frac{1}{n} \log J\left(\left.\underline{\varphi}\right|_{n}, u\right)-3 \bar{m} \epsilon_{0}-\frac{1}{n} \mathbf{C}_{0}+\mathbf{c}_{0} .
$$

Proof. The set $\mathbf{B}_{\mathrm{A}}^{s}(\underline{\varphi}, u, \eta, n)$ is empty if $(\underline{\varphi}, u) \notin \mathrm{A}$. Otherwise, by definition and Lemma $3.4 \mathrm{iv}), \mathbf{B}_{\mathrm{A}}^{s}(\underline{\varphi}, u, \eta, n)$ is contained in the set of vectors $e \in T_{u} \bar{W}^{s}(u)$ such that

$$
\begin{gathered}
\|e\|_{(\underline{\varphi}, u), 0}^{\prime}<\eta \kappa^{-1}((\underline{\varphi}, u), 0),\left\|\left.\mathbf{F}_{(\underline{\varphi}, u)}\right|_{n}(e)\right\|_{(\underline{\varphi}, u), n}^{\prime \prime}<\eta \kappa^{-1}\left(\tau^{\mathrm{N}_{n}^{\mathrm{A}}}(\underline{\varphi}, u), n-\mathrm{N}_{n}^{\mathrm{A}}\right) \text { and } \\
\left|\operatorname{Det}^{\prime} D_{e} \mathbf{F}_{(\underline{\varphi}, u), k}\right| \geqslant\left|\operatorname{Det}^{\prime} D_{0} \mathbf{F}_{(\underline{\varphi}, u), k}\right|(1-\epsilon(\underline{\varphi}, u))^{\bar{m}} \text { for } 1 \leqslant k \leqslant n,
\end{gathered}
$$


where Det $^{\prime}$ is the determinant of a linear mapping in the metrics $\|\cdot\|_{\tau^{\mathrm{N}_{k}^{\mathrm{A}}}(\underline{\varphi}, u), k-\mathrm{N}_{k}^{\mathrm{A}}}^{\prime}$ and $\|\cdot\|_{\tau^{\mathrm{N} A}(\underline{\varphi}, u), k+1-\mathrm{N}_{k}^{\mathrm{A}}}^{\prime}$. By construction, $\tau^{\mathrm{N}_{k}^{\mathrm{A}}}(\underline{\varphi}, u) \in \mathrm{A}$ and $\kappa\left(\tau^{\mathrm{N}_{k}^{\mathrm{A}}}(\underline{\varphi}, u), 0\right) \leqslant\left(\frac{\eta}{l} \kappa_{0}\right)^{1 / 2}$. Assume $\tau^{k+1}(\underline{\varphi}, u) \in$ A, i.e., $\mathrm{N}_{k+1}^{\mathrm{A}}=k+1$. Then

$$
\begin{aligned}
\|\cdot\|_{\tau^{\mathrm{N}_{k}^{\mathrm{A}}}(\underline{\varphi}, u), k+1-\mathrm{N}_{k}^{\mathrm{A}}}^{\prime} & \leqslant e^{\left(\mathrm{N}_{k+1}^{\mathrm{A}}-\mathrm{N}_{k}^{\mathrm{A}}\right) \epsilon} \kappa\left(\tau^{\mathrm{N}_{k}^{\mathrm{A}}}(\underline{\varphi}, u), 0\right) \kappa_{0}^{-1}\|\cdot\|_{\tau^{k+1}(\underline{\varphi}, u), 0}^{\prime} \\
& \leqslant e^{\left(\mathrm{N}_{k+1}^{\mathrm{A}}-\mathrm{N}_{k}^{\mathrm{A}}\right) \epsilon}\left(\frac{\eta}{l \kappa_{0}}\right)^{\frac{1}{2}}\|\cdot\|_{\tau^{k+1}}^{\prime}(\underline{\varphi}, u), 0 .
\end{aligned}
$$

By chopping $\left.\varphi\right|_{n}(u)$ into pieces in between returning times and using (3.10), (3.11), we see that there exists a geometric constant $C$ such that the set $\exp _{u} \mathbf{B}_{\mathrm{A}}^{s}(\underline{\varphi}, u, \eta, n)$ is contained in the set $\underline{\mathbf{B}}_{\mathrm{A}}^{s}(\varphi, u, \eta, n)$ of points $w \in \bar{W}^{s}(u)$ such that

$$
\begin{gathered}
d_{\bar{W}^{s}(u)}(w, u)<C \kappa_{0}^{-1} \eta \kappa^{-1}(\underline{\varphi}, u), d_{\bar{W}^{s}\left(\left.\underline{\varphi}\right|_{n} u\right)}\left(\left.\underline{\varphi}\right|_{n} w,\left.\underline{\varphi}\right|_{n} u\right)<C \kappa_{0}^{-1} \eta \kappa^{-1}\left(\tau^{\mathrm{N}_{n}^{\mathrm{A}}}(\underline{\varphi}, u), n-\mathrm{N}_{n}^{\mathrm{A}}\right) \\
\text { and } J\left(\left.\underline{\varphi}\right|_{n}, w\right) \geqslant\left(C^{-1} \kappa_{0}\right)^{\bar{m}}(1-\epsilon(\underline{\varphi}, u))^{n \bar{m}} J\left(\left.\underline{\varphi}\right|_{n}, u\right) e^{-n \bar{m} \epsilon}\left(\frac{l \kappa_{0}}{\eta}\right)^{\frac{1}{2} \bar{m} \#\left\{\mathrm{~N}_{k}^{\mathrm{A}}(\underline{\varphi}, u): k \leqslant n\right\}} .
\end{gathered}
$$

It follows that, denoting $\bar{\lambda}_{u}^{s}$ the Lebesgue measure on $\bar{W}^{s}(u)$,

$$
\overline{\mathbf{m}}_{\rho, u}^{s}\left(\exp _{u}\left(\mathbf{B}_{\mathrm{A}}^{s}(\underline{\varphi}, u, \eta, n)\right)\right) \leqslant \frac{e^{C^{\prime}}}{\bar{\lambda}_{u}^{s}\left(\mathcal{R}\left(\left.\underline{\varphi}\right|_{n}(u)\right)\right)} \int_{\left.\underline{\varphi}\right|_{n}\left(\underline{\mathbf{B}}_{\mathrm{A}}^{s}(\underline{\varphi}, u, \eta, n)\right)} J^{-1}\left(\left.\underline{\varphi}\right|_{n}, w\right) d \bar{\lambda}_{\left.\underline{\varphi}\right|_{n} u}^{s}(w),
$$

where $C^{\prime}$ is a positive constant taking into account the regularity of the density for a fixed $\rho$. It follows that, with our definition of $\mathbf{c}_{0}$,

$$
\begin{aligned}
& \overline{\mathbf{m}}_{\rho, u}^{s}\left(\exp _{u}\left(\mathbf{B}_{\mathrm{A}}^{s}(\underline{\varphi}, u, \eta, n)\right)\right) \\
& \quad \leqslant \frac{e^{C^{\prime}}}{\bar{\lambda}_{u}^{s}\left(\mathcal{R}\left(\left.\underline{\varphi}\right|_{n}(u)\right)\right)} J^{-1}\left(\left.\underline{\varphi}\right|_{n}, u\right)\left(C \kappa_{0}^{-1}\right)^{\bar{m}}(1-\epsilon(\underline{\varphi}, u))^{-n \bar{m}} e^{n \bar{m} \epsilon} e^{-\mathbf{c}_{0} \#\left\{\mathbb{N}_{k}^{\mathrm{A}}(\underline{\varphi}, u): k \leqslant n\right\}} .
\end{aligned}
$$

Note that the partition $\mathcal{R}$ is such that each element contains a ball with radius greater than some positive constant (see Section 4 ), we obtain some constant $\mathbf{C}_{0}>0$ such that

$$
-\frac{1}{n} \log \overline{\mathbf{m}}_{\rho, u}^{s}\left(\exp _{u}\left(\mathbf{B}_{\mathrm{A}}^{s}(\underline{\varphi}, u, \eta, n)\right)\right) \geqslant \frac{1}{n} \log J\left(\left.\underline{\varphi}\right|_{n}, u\right)+\bar{m} \log (1-\epsilon(\underline{\varphi}, u))-\bar{m} \epsilon-\frac{1}{n} \mathbf{C}_{0}+\mathbf{c}_{0} .
$$

The estimation in (3.9) follows for $\epsilon_{0}$ small enough.

By (3.5) and Lemma 3.4 i), we have

$$
\mathbf{c}_{0}=\frac{1}{2} \bar{m} \log \frac{l \kappa_{0}}{\eta} \leqslant \bar{m} \log \frac{\kappa_{0}}{\min _{(\underline{\varphi}, u) \in \mathrm{A}} \kappa((\underline{\varphi}, u), 0)} \leqslant 0 .
$$

So the estimation in (3.9) might be too coarse since $\mathbf{c}_{0}$ is not a priori small compared with $\epsilon_{0}$. But $\mathbf{c}_{0}$ remains unchanged when we consider (3.9) for Bowen balls for any power of $\tau$, hence it will not enter the lower bound estimation of entropy in (3.2). 
More precisely, let $M \in \mathbb{N}$ be fixed. For $\underline{\varphi} \in \mathcal{S}$, write

$$
\underline{\varphi}^{\prime}:=\left(\varphi_{0}^{\prime}, \varphi_{1}^{\prime}, \cdots, \varphi_{k}^{\prime}, \cdots\right), \text { where } \varphi_{k}^{\prime}:=\left.\underline{\varphi} \circ \sigma^{k \mathrm{M}}\right|_{\mathrm{M}}
$$

Let

$$
\sigma^{\prime}\left(\underline{\varphi}^{\prime}\right):=\left(\varphi_{1}^{\prime}, \cdots, \varphi_{k}^{\prime}, \cdots\right) \text { and } \tau^{\prime}\left(\underline{\varphi}^{\prime}, u\right):=\left(\sigma^{\prime}(\underline{\varphi}), \varphi_{0}^{\prime} u\right) .
$$

The transformation $\tau^{\prime}$ can be identified with $\tau^{\mathrm{M}}$. We can use the same $\kappa_{0}, \kappa$ for $\tau^{\prime}$ as for $\tau$, but now $\epsilon$ in (3.3) has to be changed into $\mathrm{M} \epsilon$. So we have to choose $\epsilon^{\prime}$ so that $\mathrm{M} \epsilon^{\prime}<\epsilon_{0}$. Choose $\eta^{\prime}<\eta$ small enough that, if $l^{\prime}:=l \frac{\eta^{\prime}}{\eta}$, the measurable set

$$
\mathrm{A}^{\prime}:=\mathrm{A}\left(b, l^{\prime}\right) \cap\left\{(\underline{\varphi}, u): \eta^{\prime}<\epsilon^{\prime}(\underline{\varphi}, u)\right\}
$$

has $\mu_{\rho}$ measure greater than $1-a$.

For $\mu_{\rho}$ almost all $(\underline{\varphi}, u)=\left(\underline{\varphi^{\prime}}, u\right) \in \mathrm{A}^{\prime}$ and $k \in \mathbb{N}$, let $\mathrm{M}_{k}^{\mathrm{A}^{\prime}}$ denote the last non-negative time before or equal to $k$ such that $\left(\tau^{\prime}\right)^{\mathrm{M}_{k}^{\mathrm{A}^{\prime}}}\left(\underline{\varphi^{\prime}}, u\right) \in \mathrm{A}^{\prime}$. Similar to (3.7) and (3.8), we define

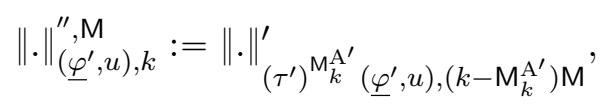

and for $\eta^{\prime}>0,(\underline{\varphi}, u) \in \mathrm{A}^{\prime}$ such that $\eta^{\prime}<\epsilon^{\prime}(\underline{\varphi}, u)$, and $n \in \mathbb{N}$, we define the modified random $\overline{\mathcal{W}}^{s}$-Bowen ball for $\tau^{\prime}$ (with respect to $\mathrm{A}^{\prime}$ ) by

$\mathbf{B}_{\mathrm{A}^{\prime}}^{s, \mathrm{M}}\left(\underline{\varphi}^{\prime}, u, \eta, n\right):=\left\{e \in T_{u} \bar{W}^{s}(u):\|e\|_{\left(\underline{\varphi}^{\prime}, u\right), 0}^{\prime \prime}<\eta^{\prime} \kappa^{-1}\left(\left(\underline{\varphi}^{\prime}, u\right), 0\right)\right.$, and for $k, 1 \leqslant k \leqslant n$,

$$
\left.\left\|\left.\mathbf{F}_{(\underline{\varphi}, u)}\right|_{k \mathrm{M}}(e)\right\|_{\left(\underline{\varphi^{\prime}}, u\right), k}^{\prime \prime}, \mathrm{M} \quad \eta \kappa^{-1}\left(\left(\tau^{\prime}\right)^{\mathrm{M}_{k}^{\mathrm{A}^{\prime}}}\left(\underline{\varphi^{\prime}}, u\right),\left(k-\mathrm{M}_{k}^{\mathrm{A}^{\prime}}\right) \mathrm{M}\right)\right\} .
$$

Then following the argument in Lemma 3.5, we obtain (observe that, by our choice of $l^{\prime}$, $\mathbf{c}_{0}=\frac{1}{2} \bar{m} \log \frac{l \kappa_{0}}{\eta}=\frac{1}{2} \bar{m} \log \frac{l^{\prime} \kappa_{0}}{\eta^{\prime}}$ has the same value as in Lemma 3.5)

Lemma 3.6. Let $-\infty<\rho<0, a \in(0,1), \mathrm{M} \in \mathbb{N}$ and $\epsilon_{0}$ be fixed. Let $\eta^{\prime}, \epsilon^{\prime}, \mathrm{A}^{\prime}$ be as in (3.12) and let $\mathbf{c}_{0}, \mathbf{C}_{0}$ be as in Lemma 3.5. Then, for $\mu_{\rho}$ almost all $(\underline{\varphi}, u) \in \mathrm{A}^{\prime}$ and all $n \in \mathbb{N}$,

(3.13) $-\frac{1}{n} \log \overline{\mathbf{m}}_{\rho, u}^{s}\left(\exp _{u}\left(\mathbf{B}_{\mathrm{A}^{\prime}}^{s, \mathrm{M}}\left(\underline{\varphi^{\prime}}, u, \eta, n\right)\right)\right) \geqslant-\frac{1}{n} \log J\left(\left.\underline{\varphi}\right|_{n \mathrm{M}}, u\right)-3 \bar{m} \mathrm{M} \epsilon_{0}-\frac{1}{n} \mathbf{C}_{0}+\mathbf{c}_{0}$.

Following Mañé ([Man83]) (see also [Thi92]), we can proceed to find partitions which have local entropy lower bound as in (3.13) in our non-invertible random setting.

Lemma 3.7. Let $\epsilon_{0}>0,-\infty<\rho<0, \mathrm{M} \in \mathbb{N}$ be fixed. Let $\eta^{\prime}, \epsilon^{\prime}, \mathrm{A}^{\prime}$ be as in (3.12) and let $\mathbf{c}_{0}, \mathbf{C}_{0}$ be as in Lemma 3.5. There exists a countable partition $\mathcal{Q}$ of $\mathcal{T}$ with $-\int \log \left(\overline{\mathbf{m}}_{\rho}(\mathcal{Q})\right) d \mu_{\rho}<+\infty$ such that for $\mu_{\rho}$ almost all $(\underline{\varphi}, u) \in \mathrm{A}^{\prime}$, we have $0<\eta^{\prime}<$ $\epsilon^{\prime}(\underline{\varphi}, u)<\epsilon_{0} / \mathrm{M}$ and

$$
\mathcal{Q}_{\mathrm{M},-n}(\underline{\varphi}, u) \subset \exp _{u}\left(\mathbf{B}_{\mathrm{A}^{\prime}}^{s, \mathrm{M}}\left(\underline{\varphi^{\prime}}, u, \eta^{\prime}, n\right)\right),
$$


where $\mathcal{Q}_{\mathrm{M},-n}:=\mathcal{Q} \bigvee\left(\tau^{\mathrm{M}}\right)^{-1} \mathcal{Q} \bigvee \cdots \bigvee\left(\tau^{\mathrm{M}}\right)^{-(n-1)} \mathcal{Q}$. Consequently, for $\mu_{\rho}$ almost all $(\underline{\varphi}, u) \in$ $\mathrm{A}^{\prime}$,

$$
\liminf _{n \rightarrow+\infty}-\frac{1}{n} \log \overline{\mathbf{m}}_{\rho, u}^{s}\left(\mathcal{Q}_{\mathrm{M},-n}(\underline{\varphi}, u)\right) \geqslant-\frac{1}{n} \log J\left(\left.\underline{\varphi}\right|_{n \mathrm{M}}, u\right)-3 \bar{m} \mathrm{M} \epsilon_{0}-\frac{1}{n} \mathbf{C}_{0}+\mathbf{c}_{0}
$$

Proof. Clearly, (3.15) is a consequence of (3.14) and (3.13). Hence, it suffices to show (3.14). Let $\eta^{\prime}, \epsilon^{\prime}, \mathrm{A}^{\prime}$ be as in (3.12). For $(\underline{\varphi}, u) \in \mathrm{A}^{\prime}, \kappa_{1}>0$ and $n \in \mathbb{N}$, set

$$
\begin{aligned}
& B_{\mathrm{A}^{\prime}}^{s, \mathrm{M}, \kappa_{1}, \kappa}\left(\underline{\varphi}^{\prime}, u, \eta^{\prime}, n\right):= \\
& \left\{w \in \bar{W}^{s}(u): d\left(\left.\underline{\varphi}\right|_{k \mathrm{M}}(w),\left.\underline{\varphi}\right|_{k \mathrm{M}}(u)\right)<\eta^{\prime} \kappa_{1}\left(\kappa\left(\left(\tau^{\prime}\right)^{\mathrm{M}_{k}^{\mathrm{A}^{\prime}}}\left(\underline{\varphi^{\prime}}, u\right),\left(k-\mathrm{M}_{k}^{\mathrm{A}^{\prime}}\right) \mathrm{M}\right)\right)^{-2}, \forall 0 \leqslant k \leqslant n\right\} .
\end{aligned}
$$

By Lemma $3.4 \mathrm{i}$ ), we see that there exists some constant $\kappa_{1}$ depending on the geometry of $(M, g)$ such that, for almost all $(\underline{\varphi}, u) \in \mathrm{A}^{\prime}$ and all $n \in \mathbb{N} \cup\{0\}$,

$$
B_{\mathrm{A}^{\prime}}^{s, \mathrm{M}, \kappa_{1}, \kappa}\left(\underline{\varphi^{\prime}}, u, \eta^{\prime}, n\right) \subset \exp _{u}\left(\mathbf{B}_{\mathrm{A}^{\prime}}^{s, \mathrm{M}}\left(\underline{\varphi}^{\prime}, u, \eta^{\prime}, n\right)\right) .
$$

Hence, to find a countable partition $\mathcal{Q}$ satisfying (3.14), it suffices to find a $\mathcal{Q}$ such that

$$
\mathcal{Q}_{\mathrm{M},-n}(\underline{\varphi}, u) \subset B_{\mathrm{A}^{\prime}}^{s, \kappa_{1}, \kappa}\left(\underline{\varphi^{\prime}}, u, \eta^{\prime}, n\right) .
$$

For each $n \in \mathbb{N} \cup\{0\}$, let $\mathrm{A}^{\prime}{ }_{n} \subset \mathrm{A}^{\prime}$ be the collection of points with $n$ as the first return time to $\mathrm{A}^{\prime}$ with respect to the map $\tau^{\mathrm{M}}$. Recall that the local stable leaf $\bar{W}_{l o c, \epsilon_{0}}^{s}(u)=\left\{w \in \bar{W}^{s}(u)\right.$ : $\left.d_{\bar{W}^{s}}(w, u)<\epsilon_{0}\right\}$ depends continuously on $u$ and for each $n$, we can choose in a continuous way a maximal $\left(4\left(l^{\prime}\right)^{2} b\right)^{-1} e^{-2\left(L+\epsilon_{0}\right) n \mathrm{M}}$ separated set in $\bar{W}_{l o c, \epsilon_{0}}^{s}(u)$. The cardinality $\mathcal{C}_{n}$ of such sets satisfies $\mathcal{C}_{n} \leqslant K^{n \mathrm{M}}$ for some $K$. Using these points, we can further slice $\mathrm{A}^{\prime}{ }_{n}$ into $\left\{\mathrm{A}_{n, \ell}^{\prime}\right\}_{\ell \leqslant \mathcal{C}_{n}}$ such that for all $(\underline{\varphi}, u) \in \mathrm{A}_{n, \ell}^{\prime}$, the intersection $\left\{w:(\underline{\varphi}, w) \in \mathrm{A}_{n, \ell}^{\prime}\right\} \cap \bar{W}_{l o c, \epsilon_{0}}^{s}(u)$ has diameter less than $\left(2\left(l^{\prime}\right)^{2} b\right)^{-1} e^{-2\left(L+\epsilon_{0}\right) n \mathrm{M}}$. The partition $\mathcal{Q}$ can be chosen to be

$$
\left\{\mathrm{A}^{\prime}{ }_{n, \ell}, n \in \mathbb{N} \cup\{0\}, \ell \leqslant \mathcal{C}_{n}, \mathcal{T} \backslash \mathrm{A}^{\prime}\right\} .
$$

Following [Man83], one checks that $\mathcal{Q}$ satisfies $-\int \log \left(\overline{\mathbf{m}}_{\rho}(\mathcal{Q})\right) d \mu_{\rho}<+\infty$ and (3.14).

Proof of Proposition 3.1. Let $-\infty<\rho<0$ be fixed. In the following, we show, for every $\epsilon_{0}>0$, there exists a finite measurable partition $\mathcal{P}$ of $\mathcal{T}$ satisfying

$$
\underline{h}_{\rho, \mathcal{P}}^{s} \geqslant \int \log J(\varphi, u) d \nu_{\rho}(\varphi) d \overline{\mathbf{m}}_{\rho}(u)-5 \bar{m} \epsilon_{0}
$$

Then, by definition of $h_{\rho}^{s}$ and (3.16),

$$
h_{\rho}^{s} \geqslant \underline{h}_{\rho, \mathcal{P}}^{s} \geqslant \int \log J(\varphi, u) d \nu_{\rho}(\varphi) d \overline{\mathbf{m}}_{\rho}(u)-5 \bar{m} \epsilon_{0}
$$

This concludes the proof of Proposition 3.1 since $\epsilon_{0}$ is arbitrary. 
Let $\mathrm{M}$ be such that $\left|\mathbf{c}_{0}\right|<\mathrm{M} \epsilon_{0}$. Let $a>0$ be small and let $\mathrm{A}^{\prime}$ and $\mathcal{Q}$ be as in Lemma 3.7. Then for $\mu_{\rho}$ almost all $(\underline{\varphi}, u) \in \mathrm{A}^{\prime},(3.15)$ holds true. Set

$$
\underline{h}_{\rho, \mathcal{Q}}^{s, \mathrm{M}}:=\liminf _{n \rightarrow+\infty}-\frac{1}{n} \int \log \overline{\mathbf{m}}_{\rho, u}^{s}\left(\mathcal{Q}_{\mathrm{M},-n}(\underline{\varphi}, u)\right) d \mu_{\rho}(\underline{\varphi}, u)
$$

For any $\alpha>0$, by our choice of $\mathcal{R}$ in Section 4 , it is true that (see Proposition 4.3)

$$
\underline{h}_{\rho, \mathcal{Q}}^{s} \geqslant \frac{1}{\mathrm{M}} \underline{h}_{\rho, \mathcal{Q}}^{s, \mathrm{M}}-\alpha \text {. }
$$

Hence, by Fatou Lemma,

$$
\underline{h}_{\rho, \mathcal{Q}}^{s} \geqslant \int_{\mathrm{A}} \liminf _{n \rightarrow+\infty}-\frac{1}{n \mathrm{M}} \log \overline{\mathbf{m}}_{\rho, u}^{s}\left(\mathcal{Q}_{\mathrm{M},-n}(\underline{\varphi}, u)\right) d \mu_{\rho}(\underline{\varphi}, u)-\alpha .
$$

Since the function $\log J(\varphi, u)$ is integrable and $\left|\mathbf{c}_{0}\right|<M \epsilon_{0}$, by using(3.15), we obtain, for $a, \alpha>0$ small,

$$
\underline{h}_{\rho, \mathcal{Q}}^{s} \geqslant \int \log J(\varphi, u) d \nu_{\rho}(\varphi) d \overline{\mathbf{m}}_{\rho}(u)-4 \bar{m} \epsilon_{0}
$$

Note that $\mathcal{Q}$ is such that $-\int \log \left(\overline{\mathbf{m}}_{\rho}(\mathcal{Q})\right) d \mu_{\rho}<+\infty$ and for any finite partition $\mathcal{P}$ such that $\mathcal{Q}$ is finer than it,

$$
\begin{aligned}
\underline{h}_{\rho, \mathcal{Q}}^{s}-\underline{h}_{\rho, \mathcal{P}}^{s} & \leqslant \limsup _{n \rightarrow+\infty} \frac{1}{n} \int H_{\overline{\mathbf{m}}_{\rho}^{s}}\left(\mathcal{Q}_{-n} \mid \mathcal{P}_{-n}\right) d \mu_{\rho} \\
& \leqslant \limsup _{n \rightarrow+\infty} \frac{1}{n} \int H_{\overline{\mathbf{m}}_{\rho}}\left(\mathcal{Q}_{-n} \mid \mathcal{P}_{-n}\right) d \mu_{\rho} \leqslant \int H_{\overline{\mathbf{m}}_{\rho}}(\mathcal{Q} \mid \mathcal{P}) d \mu_{\rho} .
\end{aligned}
$$

We can group the tail elements in $\mathcal{Q}$ together with some care to obtain a finite partition $\mathcal{P}$ satisfying the requirement in (3.16).

\section{The proof of Proposition 3.2}

Let $\overline{\mathbf{m}}$ be as in Proposition 3.2. To compare $h_{\frac{\mathbf{m}}{\mathbf{m}}}^{s}$ with $h_{\rho_{p}}^{s}$, we first formulate the entropy $\underline{h}_{\rho_{p}, \mathcal{P}}^{s}$ (see (3.1)) in terms of some conditional entropy for the unconditional measure $\mu_{\rho}$.

Let $\mathcal{W}$ be a lamination of a compact metric space. A measurable partition is said to be subordinated to $\mathcal{W}$ if its elements are bounded subsets of the leaves of $\mathcal{W}$ with nonempty interiors in the topology of the leaf. We can construct a partition $\mathcal{R}$ subordinated to $\overline{\mathcal{W}}^{s}$ by choosing a finite partition $\mathcal{X}$ of $\mathcal{O}^{s}(S M)$ into sufficiently small sets with non-empty interiors and subdivide each element of $\mathcal{X}$ into the connected components of its intersection with the leaves. We may assume $\mathcal{R}$ is such that each element contains a ball with radius greater than some positive constant. The partition $\mathcal{R}$ is measurable if it is constructed as an intersection of an increasing family $\mathcal{R}^{j}, j \in \mathbb{N}$, of finite partitions into measurable sets.

Let $\mathcal{P}$ be a finite partition of $\mathcal{O}^{s}(S M)$ and we assume that we have chosen $\mathcal{X}, \mathcal{R}=\bigvee_{j} \mathcal{R}^{j}$ as above and that $\mathcal{P}$ refines $\mathcal{X}$. We may assume that the boundaries of the elements of 
$\mathcal{P}, \mathcal{X}$ and $\mathcal{R}^{j}$ are all $\overline{\mathbf{m}}$-negligible. The conditional measures $\overline{\mathbf{m}}_{\rho, u}^{s}$ in the definition of $\underline{h}_{\rho, \mathcal{P}}^{s}$ can be taken on any measurable finite partition $\mathcal{R}$ chosen in the above way, so that

$$
\underline{h}_{\rho, \mathcal{P}}^{s}=\liminf _{n \rightarrow+\infty}-\frac{1}{n} \int \log \overline{\mathbf{m}}_{\rho, u}^{s}\left(\mathcal{P}_{-n}(\underline{\varphi}, u)\right) d \mu_{\rho}(\underline{\varphi}, u)=\liminf _{n \rightarrow+\infty} \frac{1}{n} H_{\mu_{\rho}}\left(\mathcal{P}_{-n} \mid \mathcal{R}\right) .
$$

Proving Proposition 3.2 amounts to proving that, if $\rho_{p} \rightarrow-\infty$ and $\overline{\mathbf{m}}_{\rho_{p}} \rightarrow \overline{\mathbf{m}}$ as $p \rightarrow+\infty$, then

$$
h_{\overline{\mathbf{m}}}^{s} \geqslant \limsup _{p \rightarrow+\infty} \sup _{\mathcal{P}} \liminf _{n \rightarrow+\infty} \frac{1}{n} H_{\mu_{\rho_{p}}}\left(\mathcal{P}_{-n} \mid \mathcal{R}\right) .
$$

This is true, if we can show, for any $\alpha>0$, there are partitions $\mathcal{P}, \mathcal{R}$ and $n$ large, such that for all $p$ large enough,

$$
h_{\overline{\mathbf{m}}}^{s} \geqslant \frac{1}{n} H_{\mu_{\rho_{p}}}\left(\mathcal{P}_{-n} \mid \mathcal{R}\right)-2 \alpha \geqslant \underline{h}_{\rho_{p}, \mathcal{P}}^{s}-3 \alpha \geqslant h_{\rho_{p}}^{s}-5 \alpha .
$$

The first inequality in (4.1) can be achieved if we can find $\operatorname{good} \mathcal{P}, \mathcal{R}$ for $\overline{\mathbf{m}}$ with $h_{-\infty, \mathcal{P}}^{s}$ being close to $h_{\mathbf{m}}^{s}$. So we will show the other two inequalities in (4.1) first.

We begin with the second inequality in (4.1), which is not trivial in our setting since the conditional entropy sequence $H_{\mu_{\rho_{p}}}\left(\mathcal{P}_{-n} \mid \mathcal{R}\right)$ is not necessarily a subadditive sequence in $n$.

Lemma 4.1. Given $\mathcal{X}, \mathcal{R}$ and $\mathcal{P}$ as above, there exists a countable partition $\mathcal{Q}$ of $\mathcal{T}$ such that the partition $\mathcal{R} \bigvee \tau^{-1} \mathcal{P} \bigvee \tau^{-1} \mathcal{Q}$ is finer than $\tau^{-1} \mathcal{R}$. Moreover, given $\alpha>0$, there are $\delta$ and $\Lambda$ such that if the diameters of the elements of $\mathcal{X}$ are smaller than $\delta$ and if $\rho<\Lambda$, one can choose $\mathcal{Q}$ with $H_{\mu_{\rho}}(\mathcal{Q})<\alpha$.

Proof. For $u, w \in \mathcal{O}^{s}(S M)$ in the same $\overline{\mathcal{W}}^{s}$ leaf, write $d^{s}(u, w)$ for the distance between $u$ and $w$ along their common leaf. For any $\delta>0$, there are two constants $c(\delta)$ and $C(\delta)$ such that if $u$ and $w$ are on the same leaf and $d(u, w)<\delta$, then either $d^{s}(u, w)<c(\delta)$ or $d^{s}(u, w) \geqslant C(\delta)$. We can ensure that $c(\delta) \rightarrow 0$ as $\delta \rightarrow 0$ and that $C(\delta) \rightarrow+\infty$ as $\delta \rightarrow 0$. Suppose $u$ and $w$ are in the same element of the partition $\mathcal{R}$ and that $\varphi_{0} u$ and $\varphi_{0} w$ are in the same element of $\mathcal{X}$. If $d^{s}\left(\varphi_{0} u, \varphi_{0} w\right)<C(\delta)$, in particular, as soon as $d^{s}(u, w)<C(\delta) /\left\|\varphi_{0}\right\|_{C^{1}}$, then $\varphi_{0} u$ and $\varphi_{0} w$ are in the same connected component of $\overline{\mathcal{W}}^{s}$ and thus in the same element of $\mathcal{R}$.

To obtain Lemma 4.1, it is therefore enough to take the partition $\mathcal{Q}$ of $\mathcal{T}$ as follows: the projection on $\mathcal{S}$ depends only on the first coordinate $\varphi_{0}$ and is the partition $A_{n}, n \geqslant 0$, where $A_{n}:=\left\{\varphi_{0}: n C(\delta) \leqslant\left\|\varphi_{0}\right\|_{C^{1}} \leqslant(n+1) C(\delta)\right\} ; A_{0} \times \mathcal{O}^{s}(S M)$ is one element of $\mathcal{Q}$; on each $A_{n}, n>0$, we further cut $\mathcal{O}^{s} S M$ into $N_{n}$ pieces of diameter smaller than $1 /(n+1)$.

The entropy of $\mathcal{Q}$ satisfies

$$
H_{\mu_{\rho}}(\mathcal{Q}) \leqslant H_{\mu_{\rho}}\left(\left\{A_{n}: n \geqslant 0\right\}\right)+\mathbf{c} \sum_{n=1}^{\infty} \nu_{\rho}\left(A_{n}\right) \log n,
$$

where $\mathbf{c}$ is some constant depending on the geometry of $\overline{\mathcal{W}}^{s}$. Given $\alpha>0$, we will have $H_{\mu_{\rho}}(\mathcal{Q})<\alpha$ as soon as $\nu_{\rho}\left(\left\{\varphi:\|\varphi\|_{C^{1}}>C(\delta)\right\}\right)$ and the integral $\int_{\left\{\varphi:\|\varphi\|_{C^{1}}>C(\delta)\right\}} \log \|\varphi\|_{C^{1}} d \nu_{\rho}$ 
are sufficiently small. These two conditions can be realized by choosing $\delta$ small and $\rho$ close enough to $-\infty$.

Proposition 4.2. Given $\alpha>0$, there is $\delta>0$ and $\Lambda$ such that, for all $n>0$, if the diameter of the elements of $\mathcal{X}$ are smaller than $\delta$ and $\rho<\Lambda$,

$$
\frac{1}{n} H_{\mu_{\rho}}\left(\mathcal{P}_{-n} \mid \mathcal{R}\right) \geqslant \liminf _{n \rightarrow+\infty} \frac{1}{n} H_{\mu_{\rho}}\left(\mathcal{P}_{-n} \mid \mathcal{R}\right)-\alpha=\underline{h}_{\rho, \mathcal{P}}^{s}-\alpha
$$

Proof. Let $\mathcal{Q}$ be as in Lemma 4.1. Then we have that the mapping $n \mapsto H_{\mu_{\rho}}\left(\mathcal{P}_{-n} \bigvee \mathcal{Q}_{-n} \mid \mathcal{R}\right)$ is subadditive. Indeed, for $n, n^{\prime} \in \mathbb{N}$,

$$
\begin{aligned}
H_{\mu_{\rho}}\left(\mathcal{P}_{-\left(n+n^{\prime}\right)} \bigvee \mathcal{Q}_{-\left(n+n^{\prime}\right)} \mid \mathcal{R}\right)= & H_{\mu_{\rho}}\left(\mathcal{P}_{-n} \bigvee \mathcal{Q}_{-n} \mid \mathcal{R}\right) \\
& +H_{\mu_{\rho}}\left(\mathcal{P}_{-\left(n+n^{\prime}\right)}^{-n} \bigvee \mathcal{Q}_{-\left(n+n^{\prime}\right)}^{-n} \mid \mathcal{R} \bigvee \mathcal{P}_{-n} \bigvee \mathcal{Q}_{-n}\right)
\end{aligned}
$$

where $\mathcal{P}_{-\left(n+n^{\prime}\right)}^{-n}:=\tau^{-n} \mathcal{P} \bigvee \cdots \bigvee \tau^{-\left(n+n^{\prime}-1\right)} \mathcal{P}$ and $\mathcal{Q}_{-\left(n+n^{\prime}\right)}^{-n}$ is defined in the same way. Moreover, by Lemma 4.1, the partition $\mathcal{R} \bigvee \mathcal{P}_{-n} \bigvee \mathcal{Q}_{-n}$ is finer than $\tau^{-n} \mathcal{R}$ and the last term is smaller than $H_{\mu_{\rho}}\left(\mathcal{P}_{-\left(n+n^{\prime}\right)}^{-n} \bigvee \mathcal{Q}_{-\left(n+n^{\prime}\right)}^{-n} \mid \tau^{-n} \mathcal{R}\right)$. The desired subaddivity follows by invariance of $\mu_{\rho}$ under $\tau^{n}$. Hence (4.2) follows since

$$
\begin{aligned}
\liminf _{n \rightarrow+\infty} \frac{1}{n} H_{\mu_{\rho}}\left(\mathcal{P}_{-n} \mid \mathcal{R}\right) & \leqslant \liminf _{n \rightarrow+\infty} \frac{1}{n} H_{\mu_{\rho}}\left(\mathcal{P}_{-n} \bigvee \mathcal{Q}_{-n} \mid \mathcal{R}\right) \\
& =\inf _{n} \frac{1}{n} H_{\mu_{\rho}}\left(\mathcal{P}_{-n} \bigvee \mathcal{Q}_{-n} \mid \mathcal{R}\right) \\
& \leqslant \inf _{n} \frac{1}{n} H_{\mu_{\rho}}\left(\mathcal{P}_{-n} \mid \mathcal{R}\right)+H_{\mu_{\rho}}(\mathcal{Q}) \\
& \leqslant \inf _{n} \frac{1}{n} H_{\mu_{\rho}}\left(\mathcal{P}_{-n} \mid \mathcal{R}\right)+\alpha
\end{aligned}
$$

Proposition 4.3. Let $\mathrm{M} \in \mathbb{N}$ and let $\mathcal{P}$ be as in Proposition 4.2. Then

$$
\underline{h}_{\rho, \mathcal{P}}^{s, \mathrm{M}} \leqslant \mathrm{M} \cdot\left(\underline{h}_{\rho, \mathcal{P}}^{s}+\alpha\right) .
$$

Proof. Let $\mathcal{Q}$ be as in Lemma 4.1. Recall that

$$
\underline{h}_{\rho, \mathcal{P}}^{s, \mathrm{M}}=\liminf _{n \rightarrow+\infty}-\frac{1}{n} \int \log \overline{\mathbf{m}}_{\rho, u}^{s}\left(\mathcal{P}_{\mathrm{M},-n}(\underline{\varphi}, u)\right) d \mu_{\rho}(\underline{\varphi}, u)=\liminf _{n \rightarrow+\infty} \frac{1}{n} H_{\mu_{\rho}}\left(\mathcal{P}_{\mathrm{M},-n} \mid \mathcal{R}\right) .
$$


Hence,

$$
\begin{aligned}
\underline{h}_{\rho, \mathcal{P}}^{s, \mathrm{M}} \leqslant \underline{h}_{\rho, \mathcal{P}_{-\mathrm{M}}}^{s, \mathrm{M}} & =\mathrm{M} \cdot \liminf _{n \rightarrow+\infty} \frac{1}{n \mathrm{M}} H_{\mu_{\rho}}\left(\mathcal{P}_{-\mathrm{M} n} \mid \mathcal{R}\right) \\
& \leqslant \mathrm{M} \cdot \liminf _{n \rightarrow+\infty} \frac{1}{n \mathrm{M}} H_{\mu_{\rho}}\left(\mathcal{P}_{-\mathrm{M} n} \bigvee \mathcal{Q}_{-\mathrm{M} n} \mid \mathcal{R}\right) \\
& =\mathrm{M} \cdot \liminf _{n \rightarrow+\infty} \frac{1}{n} H_{\mu_{\rho}}\left(\mathcal{P}_{-n} \bigvee \mathcal{Q}_{-n} \mid \mathcal{R}\right) \\
& \leqslant \mathrm{M} \cdot\left(\underline{h}_{\rho, \mathcal{P}}^{s}+H_{\mu_{\rho}}(\mathcal{Q})\right) \\
& \leqslant \mathrm{M} \cdot\left(\underline{h}_{\rho, \mathcal{P}}^{s}+\alpha\right) .
\end{aligned}
$$

Next we show the last inequality in (4.1). For this, we first state the results extending to our context the classical results of [Bow72], [Yue94] and [Buz97] (compare with [CY05]).

For $u \in \mathcal{O}^{s}(S M), \varphi \in \mathcal{S}, \eta>0$ and $n \in \mathbb{N}$, define the random $\overline{\mathcal{W}}^{s}$-Bowen ball by

$$
B^{s}(\underline{\varphi}, u, \eta, n):=\left\{w \in \bar{W}^{s}(u): d\left(\left.\underline{\varphi}\right|_{k}(w),\left.\underline{\varphi}\right|_{k}(u)\right)<\eta \text { for } 0 \leqslant k \leqslant n\right\} .
$$

The following notion was introduced by Bowen ([Bow72]) for a single map and by CowiesonYoung $([\mathbf{C Y 0 5}])$ in the random case. Since our mappings are smooth only along the foliation $\overline{\mathcal{W}}^{s}$, we introduce a variant by restricting to the leaves $\bar{W}^{s}$. Fix $\zeta>0$ and a sequence $\varphi \in \mathcal{S}$. We denote for $u \in \mathcal{O}^{s}(S M), \eta>0$ and $n \in \mathbb{N}, r(\zeta, \varphi, u, \eta, n)$ the smallest number of random $\overline{\mathcal{W}}^{s}$-Bowen balls $B^{s}(\varphi, w, \eta, n)$ needed to cover the random $\overline{\mathcal{W}}^{s}$-Bowen ball $B^{s}(\underline{\varphi}, u, \zeta, n)$. We then set

$$
h_{l o c}^{s}(\zeta, \underline{\varphi}):=\sup _{u \in \mathcal{O}^{s}(S M)} \lim _{\eta \rightarrow 0} \limsup _{n \rightarrow+\infty} \frac{1}{n} \log r(\zeta, \underline{\varphi}, u, \eta, n) .
$$

The function $\underline{\varphi} \mapsto h_{l o c}^{s}(\zeta, \underline{\varphi})$ is $\sigma$-invariant; we denote $h_{l o c, \rho}^{s}(\zeta)$ its $\nu_{\rho}^{\otimes \mathbb{N} \cup\{0\}}$-a.e. value.

The following three propositions (Proposition 4.4, Proposition 4.5 and Proposition 4.6) are proven in [CY05] for the global entropy with the additional hypotheses that $\nu_{\rho}$ are supported in a fixed neighborhood $\mathcal{N}$ of $\overline{\boldsymbol{\Phi}}_{-1}$ in $D^{\infty}\left(\mathcal{O}^{s} S M\right)$ and that $\nu_{\rho}$ converge to $\nu_{-\infty}$ as $\rho \rightarrow-\infty$, in the sense that any $D^{\infty}\left(\mathcal{O}^{s} S M\right)$ neighborhood of $\overline{\boldsymbol{\Phi}}_{-1}$ has eventually full measure for $\nu_{\rho}$. In our case, we have two extensions of the argument in [CY05]: one is that the distributions $\nu_{\rho}$ are not supported on a neighbourhood of $\overline{\mathbf{\Phi}}_{-1}$, but there is a tail; the other extension is that our mappings are not smooth everywhere, but only along the leaves of the foliation $\overline{\mathcal{W}}^{s}$.

Proposition 4.4. Given $\alpha>0, \zeta>0$, let $\mathcal{X}$ be as in Proposition 4.2. Assume that the diameters of the elements of $\mathcal{P} \cap \mathcal{R}$ are all smaller than $\zeta$. Then, for all $\rho$ close enough to $-\infty$,

$$
h_{\rho}^{s}-\underline{h}_{\rho, \mathcal{P}}^{s} \leqslant h_{l o c, \rho}^{s}(\zeta)+\alpha .
$$


Proof. Let $\mathrm{M}$ be a fixed positive integer and set $h_{\rho}^{s, \mathrm{M}}:=\sup _{\mathcal{P}} \underline{h}_{\rho, \mathcal{P}}^{s, \mathrm{M}}$. Since

$$
\underline{h}_{\rho, \mathcal{P}}^{s, \mathrm{M}}=\mathrm{M} \cdot \liminf _{n \rightarrow+\infty} \frac{1}{n \mathrm{M}} H_{\mu_{\rho}}\left(\mathcal{P}_{-\mathrm{M} n} \mid \mathcal{R}\right) \geqslant \mathrm{M} \cdot \underline{h}_{\rho, \mathcal{P}}^{s},
$$

we have

$$
h_{\rho}^{s, \mathrm{M}} \geqslant \sup _{\mathcal{P}} \underline{h}_{\rho, \mathcal{P}}^{s, \mathrm{M}} \geqslant \mathrm{M} \cdot \sup _{\mathcal{P}} \underline{h}_{\rho, \mathcal{P}}^{s, \mathrm{M}}=\mathrm{M} \cdot h_{\rho}^{s} .
$$

Following [Bow72, Section 3], we obtain in our random setting that there is some positive constant $\mathbf{c}$ which depends on the geometry of $\overline{\mathcal{W}}^{s}$ such that for any $\beta>0$,

$$
h_{\rho}^{s, \mathrm{M}} \leqslant \underline{h}_{\rho, \mathcal{P}}^{s, \mathrm{M}}+\mathrm{M}\left(h_{l o c, \rho}^{s}(\zeta)+\beta\right)+\mathbf{c} .
$$

Using (4.3) and (4.5), we deduce that

$$
h_{\rho}^{s} \leqslant \underline{h}_{\rho, \mathcal{P}}^{s}+h_{l o c, \rho}^{s}(\zeta)+\alpha+\beta+\frac{1}{\mathrm{M}} \mathbf{c} .
$$

Letting $\beta \rightarrow 0$ and then $\mathrm{M} \rightarrow+\infty$, we obtain the inequality (4.4).

Let $\mathrm{M}$ be a fixed positive integer. We define for $u \in \mathcal{O}^{s}(S M), \underline{\varphi} \in \mathcal{S}, \eta>0$ and $n \in \mathbb{N}$,

$$
B^{s, \mathrm{M}}(\underline{\varphi}, u, \eta, n):=\left\{w \in \bar{W}^{s}(u): d\left(\left.\underline{\varphi}\right|_{k \mathrm{M}}(w),\left.\underline{\varphi}\right|_{k \mathrm{M}}(u)\right)<\eta \text { for } 0 \leqslant k \leqslant n\right\},
$$

$r^{\mathrm{M}}(\zeta, \underline{\varphi}, u, \eta, n)$ the smallest number of $B^{s, \mathrm{M}}(\underline{\varphi}, w, \eta, n)$ balls needed to cover the $B^{s, \mathrm{M}}(\underline{\varphi}, u, \zeta, n)$ ball,

$$
h_{l o c}^{s, \mathrm{M}}(\zeta, \underline{\varphi}):=\sup _{u \in \mathcal{O}^{s}(S M)} \lim _{\eta \rightarrow 0} \limsup _{n \rightarrow+\infty} \frac{1}{n} \log r^{\mathrm{M}}(\zeta, \underline{\varphi}, u, \eta, n)
$$

and $h_{l o c, \rho}^{s, \mathrm{M}}(\zeta)$ the $\nu_{\rho}^{\otimes \mathrm{N} \cup\{0\}}$-a.e. value of $h_{l o c}^{s, \mathrm{M}}(\zeta, \underline{\varphi})$.

Proposition 4.5. With the above notations, we have, for all $\rho<0, \zeta>0$,

$$
h_{l o c, \rho}^{s}(\zeta) \leqslant \frac{1}{\mathrm{M}} h_{l o c, \rho}^{s, \mathrm{M}}(\zeta)
$$

Proof. Observe that $B^{s}(\varphi, u, \zeta, n \mathrm{M})$ is a subset of $B^{s, \mathrm{M}}(\underline{\varphi}, u, \zeta, n)$, so we are going to cover $B^{s, \mathrm{M}}(\underline{\varphi}, u, \zeta, n)$ with $B^{s}(\underline{\varphi}, w, \eta, n \mathrm{M})$ balls, $\eta$ arbitrarily small. Start with a cover of $B^{s, \mathrm{M}}(\underline{\varphi}, u, \zeta, n)$ with $B^{s, \mathrm{M}}\left(\underline{\varphi}, w_{\ell}, \eta, n\right)$ balls with $1 \leqslant \ell \leqslant r^{\mathrm{M}}(\zeta, \underline{\varphi}, u, \eta, n)$ and fix $K>0$ big. Let $\varkappa(\varphi):=\max \left\{\left\|\left.\varphi\right|_{k}\right\|_{C^{1}}: 0 \leqslant k<\mathrm{M}\right\}$. If $\varkappa\left(\sigma^{j \mathrm{M}} \varphi\right) \leqslant \bar{K}$ for all $j, 0 \leqslant j<n$, then each $\bar{B}^{s, \mathrm{M}}\left(\underline{\varphi}, w_{\ell}, \eta, n\right)$ ball is contained in $B^{s}\left(\underline{\varphi}, w_{\ell}, 2 K \eta, n \mathrm{M}\right)$ and we take these $B^{s}\left(\underline{\varphi}, w_{\ell}, 2 K \eta, n \overline{\mathrm{M}}\right)$ balls in our cover of $B^{s, \mathrm{M}}(\underline{\varphi}, u, \zeta, \bar{n})$. Otherwise, assume, for instance, that $\varkappa(\underline{\varphi})>K$, we find, for each $w_{\ell}$, at most $\bar{c}[\varkappa(\varphi) / K\rceil^{2 \bar{m}}$ points $w_{\ell^{\prime}}^{\prime}$ such that the union of the $B^{s}\left(\underline{\varphi}, w_{\ell^{\prime}}^{\prime}, 2 K \eta, \mathrm{M}\right)$ balls cover $B^{s, \mathrm{M}}\left(\underline{\varphi}, w_{\ell}, \eta, 1\right)$, where $\mathbf{c}$ is some positive constant depending on the geometry of $\overline{\mathcal{W}}^{s}$ and $\lceil a\rceil$ denotes the smallest integer greater than $a$. Working inductively, we see that

$$
r(\zeta, \underline{\varphi}, u, 2 K \eta, n \mathrm{M}) \leqslant r^{\mathrm{M}}(\zeta, \underline{\varphi}, u, \eta, n) \prod_{j=0}^{n-1}\left\lceil\varkappa\left(\sigma^{j \mathrm{M}} \underline{\varphi}\right) / K\right\rceil^{\bar{m}} \mathbf{c}^{\left.\prod_{j=0}^{n-1} \chi_{\{\varkappa(\sigma j \mathrm{M}} \underline{\varphi}>K\right\}} .
$$


It follows that for all $K>0, \underline{\varphi} \in \mathcal{S}$,

$$
\mathrm{M} h_{l o c}^{s}(\zeta, \underline{\varphi}) \leqslant h_{l o c}^{s, \mathrm{M}}(\zeta, \underline{\varphi})+\limsup _{n \rightarrow+\infty} \frac{\bar{m}}{n} \sum_{j=o}^{n-1} \log \left[\varkappa\left(\sigma^{j \mathrm{M}} \underline{\varphi}\right) / K\right\rceil+\limsup _{n \rightarrow+\infty} \frac{\log \mathbf{c}}{n} \sum_{j=o}^{n-1} \chi_{\left\{\varkappa\left(\sigma^{j \mathrm{M}} \underline{\varphi}\right)>K\right\}} .
$$

Finally, we get, for all $\rho<0$, all $\zeta>0, K>0$,

$$
\mathrm{M} h_{l o c, \rho}^{s}(\zeta) \leqslant h_{l o c, \rho}^{s, \mathrm{M}}(\zeta, \underline{\varphi})+\bar{m} \mathbb{E}[\log [\varkappa(\underline{\varphi}) / K\rceil]+\mathbb{P}[\varkappa(\underline{\varphi})>K] \log \mathbf{c} .
$$

Since $\mathbb{E}[\log \varkappa]<+\infty$, Proposition 4.5 follows by letting $K$ go to infinity.

Proposition 4.6. Fix $\zeta>0$ small and $\rho<0$. For all $r \in \mathbb{N}$, there is a positive constant $C(r)$ such that, for all $\mathrm{M} \in \mathbb{N}$,

$$
\begin{aligned}
& h_{l o c, \rho}^{s, \mathrm{M}}(\zeta) \\
& \quad \leqslant \frac{\bar{m}}{r} \int \log \left(\max \left\{\zeta^{s-1}\left\|\left.\left(\left.\varphi\right|_{\mathrm{M}}\right)\right|_{\bar{W}^{s}(u)}\right\|_{C^{s}}: 1 \leqslant s \leqslant r, u \in \mathcal{O}^{s}(S M)\right\}\right) d \nu_{\rho}^{\otimes \mathrm{M}}\left(\left.\varphi\right|_{\mathrm{M}}\right)+\log C(r) .
\end{aligned}
$$

Proof. Fix $r>0, \mathrm{M} \in \mathbb{N}$, a sequence $\varphi \in \mathcal{S}$ and $\zeta>0$. Two points $w, w^{\prime} \in \bar{W}^{s}(u)$ are said to be $(\mathrm{M}, n, \eta)$-separated if

$$
\max \left\{d\left(\left.\underline{\varphi}\right|_{k \mathrm{M}}(w),\left.\underline{\varphi}\right|_{k \mathrm{M}}\left(w^{\prime}\right)\right): 0 \leqslant k \leqslant n\right\}>\eta .
$$

It is clear that $r^{\mathrm{M}}(\zeta, \varphi, u, \eta, n)$ is bounded from above by $s^{\mathrm{M}}(\zeta, \varphi, u, \eta, n)$, the maximal

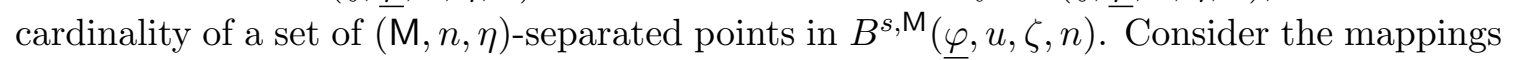
$\varphi_{k}^{\prime}=\left.\underline{\varphi}\right|_{\mathrm{M}} \circ \sigma^{k \mathrm{M}}$ and their standard magnifications ${\hat{\varphi^{\prime}}}_{k}: B(0,2)^{\bar{m}} \rightarrow \mathbb{R}^{\bar{m}}$ as explained in [CY 05], page 1129. In particular, we have $\left\|\hat{\varphi}^{\prime}{ }_{k}\right\|_{C^{s}} \leqslant \zeta^{s-1}\left\|\varphi^{\prime}{ }_{k}\right\|_{C^{s}}$. Using this, we can estimate $s^{\mathrm{M}}(\zeta, \varphi, u, \eta, n)$ by following almost verbatim the argument for the proof of Proposition 3 in [CY 05] (which is based on the 'Renormalization' Theorem in [Yom87] and a telescoping construction in [Buz97]) and obtain some constant $C_{1}(r, \bar{m}, \bar{m})=: C(r)$ as in [CY05, Theorem 4] such that

$$
\begin{aligned}
& s^{\mathrm{M}}(\zeta, \underline{\varphi}, u, \eta, n) \\
& \quad \leqslant C(r)^{n}\left(\frac{4}{\eta}\right)^{\bar{m}} \prod_{k=0}^{n-1}\left(\max \left\{\zeta^{s-1}\left\|\left.\left(\varphi_{k}^{\prime}\right)\right|_{\bar{W}^{s}(u)}\right\|_{C^{s}}: 1 \leqslant s \leqslant r, u \in \mathcal{O}^{s}(S M)\right\}\right)^{\bar{m} / r} .
\end{aligned}
$$

Since $\varphi_{k}^{\prime}$ are independent, the ergodic theorem gives Proposition 4.6.

Corollary 4.7. For any $\alpha>0$, there exists $\zeta_{0}>0$ such that if $\zeta \leqslant \zeta_{0}$, then

$$
\limsup _{\rho \rightarrow-\infty} h_{l o c, \rho}^{s}(\zeta)<\alpha .
$$


Proof. Fix $r \geqslant 2$. We choose $\mathrm{M} \in \mathbb{N}$ large such that $\frac{1}{\mathrm{M}} \log C(r) \leqslant \frac{\bar{m}}{r} B_{1,1}$, where $B_{1,1}$ is defined in (2.6). Fix $\zeta \leqslant 1, \rho<0$. By $(4.6), h_{l o c, \rho}^{s}(\zeta) \leqslant \frac{1}{\mathrm{M}} h_{l o c, \rho}^{s, \mathrm{M}}(\zeta)$. Therefore,

$h_{l o c, \rho}^{s}(\zeta) \leqslant \frac{\bar{m}}{r} B_{1,1}+\frac{\bar{m}}{r \mathrm{M}} \int \log \left(\max _{u}\left(\left\|\left.\left.\varphi\right|_{\mathrm{M}}\right|_{\bar{W}^{s}(u)}\right\|_{C^{1}}+\zeta \sum_{2 \leqslant s \leqslant r}\left\|\left.\left.\varphi\right|_{\mathrm{M}}\right|_{\bar{W}^{s}(u)}\right\|_{C^{s}}\right)\right) d \nu_{\rho}^{\otimes \mathrm{M}}\left(\left.\varphi\right|_{\mathrm{M}}\right)$.

Write, for $\alpha>0, \log ^{+} \alpha:=\max \{\log \alpha, 0\}$. We have, using $\log \left(\alpha_{1}+\alpha_{2}\right) \leqslant \log ^{+} \alpha_{1}+\alpha_{2}$, for $\alpha_{1}, \alpha_{2}>0$,

$$
\begin{aligned}
& \log \left(\max _{u}\left(\left\|\left.\left.\varphi\right|_{\mathrm{M}}\right|_{\bar{W}^{s}(u)}\right\|_{C^{1}}+\zeta \sum_{2 \leqslant s \leqslant r}\left\|\left.\left.\varphi\right|_{\mathrm{M}}\right|_{\bar{W}^{s}(u)}\right\|_{C^{s}}\right)\right) \\
& \leqslant \log ^{+}\left(\max _{u}\left(\left\|\left.\left.\varphi\right|_{\mathrm{M}}\right|_{\bar{W}^{s}(u)}\right\|_{C^{1}}\right)\right)+\zeta \sum_{2 \leqslant s \leqslant r} \max _{u}\left(\left\|\left.\left.\varphi\right|_{\mathrm{M}}\right|_{\bar{W}^{s}(u)}\right\|_{C^{s}}\right) \\
& \leqslant \sum_{k=0}^{\mathrm{M}-1} \log ^{+}\left(\max _{u}\left(\left\|\left.\varphi\right|_{\bar{W}^{s}(u)}\right\|_{C^{1}}\right) \circ \sigma^{k}\right)+\zeta \sum_{2 \leqslant s \leqslant r} \max _{u}\left(\left\|\left.\left.\varphi\right|_{\mathrm{M}}\right|_{\bar{W}^{s}(u)}\right\|_{C^{s}}\right) .
\end{aligned}
$$

We get by integrating with respect to $\nu_{\rho}^{\otimes \mathrm{M}}$,

$$
h_{l o c, \rho}^{s}(\zeta) \leqslant \frac{\bar{m}}{r} B_{1,1}+\frac{\bar{m}}{r} B_{1,1}(\rho)+\zeta \frac{\bar{m}}{r \mathrm{M}} \sum_{2 \leqslant s \leqslant r} B_{s, \mathrm{M}}(\rho),
$$

where $B_{1,1}(\rho), B_{2, \mathrm{M}}(\rho), \cdots, B_{r, \mathrm{M}}(\rho)$ are defined in (2.6). Note that, by Proposition 2.7 ii),

$$
B_{s, \mathrm{M}}=\limsup _{\rho \rightarrow-\infty} B_{s, \mathrm{M}}(\rho)<+\infty, \forall 1 \leqslant s \leqslant r
$$

hence

$$
\inf _{\zeta>0} \limsup _{\rho \rightarrow-\infty} h_{l o c, \rho}^{s}(\zeta) \leqslant \frac{2 \bar{m}}{r} \inf _{\zeta>0}\left(B_{1,1}+\zeta \frac{1}{2 \mathrm{M}} \sum_{s=2}^{r} B_{s, \mathrm{M}}\right)=\frac{2 \bar{m}}{r} B_{1,1} .
$$

Since $r$ is arbitrary, the corollary follows.

Proof of Proposition 3.2. Fix $\alpha>0$. We can choose the diameters of the elements of $\mathcal{X}$ smaller than $c \zeta_{0}$, where $c$ is a constant depending on the local geometry of the leaves so that the diameter of the elements of $\mathcal{P} \cap \mathcal{R}$ are smaller than $\zeta_{0}$ and Corollary 4.7 applies. We can also ensure that these diameters are smaller than $\delta$ given by Proposition 4.2. We may assume that the boundaries of the elements of $\mathcal{P}, \mathcal{X}$ and $\mathcal{R}^{j}$ are all $\overline{\mathbf{m}}$-negligible.

By definition, $h_{\overline{\mathbf{m}}}^{s} \geqslant \liminf _{n \rightarrow+\infty} \inf _{j} \frac{1}{n} H_{\overline{\mathbf{m}}}\left(\mathcal{P}_{-n} \mid \mathcal{R}^{j}\right)$. We can choose $n$ and $j$ so that

$$
h_{\mathbf{m}}^{s} \geqslant \frac{1}{n} H_{\overline{\mathbf{m}}}\left(\mathcal{P}_{-n} \mid \mathcal{R}^{j}\right)-\alpha .
$$

Consider now $\rho_{p}, p \in \mathbb{N}$, such that $\rho_{p} \rightarrow-\infty$ and $\overline{\mathbf{m}}_{\rho_{p}} \rightarrow \overline{\mathbf{m}}$ as $p \rightarrow+\infty$. For $\mathbb{P}$-a.e. $\omega \in \Omega$, each element of the partition $\bigcap_{k=0}^{n}\left(\left.\underline{\varphi}_{\rho_{p}}\right|_{k}(\omega)\right)^{-1} \mathcal{P}$ converge in the Hausdorff metric towards the corresponding element $\bigcap_{k=0}^{n} \overline{\mathbf{\Phi}}_{k} \mathcal{P}$. Note that all these elements of $\mathcal{P}_{-n}$, and 
the elements of $\mathcal{R}^{j}$ have $\overline{\mathbf{m}}$ negligible boundaries. It follows that there exists $P \in \mathbb{N}$ such that for $p \geqslant P$,

$$
\frac{1}{n} H_{\overline{\mathbf{m}}}\left(\mathcal{P}_{-n} \mid \mathcal{R}^{j}\right) \geqslant \frac{1}{n} H_{\mu_{\rho_{p}}}\left(\mathcal{P}_{-n} \mid \mathcal{R}^{j}\right)-\alpha \geqslant \frac{1}{n} H_{\mu_{\rho_{p}}}\left(\mathcal{P}_{-n} \mid \mathcal{R}\right)-\alpha .
$$

The second inequality holds because the partition $\mathcal{R}$ is finer than $\mathcal{R}^{j}$. By Proposition 4.2, we have, by our choice of $\delta$ and as soon as $\rho_{p}<\Lambda$,

$$
\frac{1}{n} H_{\mu_{\rho_{p}}}\left(\mathcal{P}_{-n} \mid \mathcal{R}\right) \geqslant \underline{h}_{\rho_{p}, \mathcal{P}}^{s}-\alpha \geqslant h_{\rho_{p}}^{s}-2 \alpha-h_{\text {loc, } \rho_{p}}^{s}(\zeta),
$$

where the second equality follows from Proposition 4.4. Finally, using all the above inequalities (i.e., (4.7), (4.8) and (4.9)) and Corollary 4.7, we find that

$$
h_{\overline{\mathbf{m}}}^{s} \geqslant \limsup _{p \rightarrow+\infty} h_{\rho_{p}}^{s}-5 \alpha .
$$

Proposition 3.2 follows from the arbitrariness of $\alpha$.

\section{REFERENCES}

[Bow72] R. Bowen, Entropy-expansive maps, Trans. Amer. Math. Soc. 164 (1972), 323-331.

[BB95] J. Bahnmüller and T. Bogenschütz, A Margulis-Ruelle inequality for random dynamical systems, Arch. Math. 64 (1995), no. 3, 246-253.

[BR75] R. Bowen and D. Ruelle, The ergodic theory of Axiom A flows, Invent. Math. 29 (1975), no. 3, 181-202.

[BY19] A. Blumenthal and L.-S. Young, Equivalence of physical and SRB measures in random dynamical systems, Nonlinearity 32 (2019), no. 4, 1494-1524.

[Buz97] J. Buzzi, Intrinsic ergodicity of smooth interval maps, Israel J. Math. 100 (1997), 125-161.

[CE86] A. P. Carverhill and K. D. Elworthy, Lyapunov exponents for a stochastic analogue of the geodesic flow, Trans. Amer. Math. Soc. 295 (1986), no. 1, 85-105.

[CY05] W. Cowieson and L.-S. Young, SRB measures as zero-noise limits, Ergod. Th. E3 Dynam. Sys. 25 (2005), no. 4, 1115-1138.

[EO73] P. Eberlein and B. O'Neill, Visibility manifolds, Pacific J. Math. 46 (1973), 45-109.

[Elw82] K. D. Elworthy, Stochastic Differential Equations on Manifolds, London Mathematical Society Lecture Note Series, 70. Cambridge University Press, Cambridge-New York, 1982.

[Gar83] L. Garnett, Foliations, the ergodic theorem and Brownian motion, J. Funct. Anal. 51 (1983), no. $3,285-311$.

[Ham97] U. Hamenstädt, Harmonic measures for compact negatively curved manifolds, Acta Math. 178 (1997), no. 1, 39-107.

[Kai88] V. A. Kaimanovich, Brownian motion on foliations: entropy, invariant measures, mixing, Funct. Anal. Appl. 22 (1988), no. 4, 326-328.

[Kai90] V. A. Kaimanovich, Invariant measures of the geodesic flow and measures at infinity on negatively curved manifolds, Hyperbolic behaviour of dynamical systems (Paris, 1990). Ann. Inst. H. Poincaré, Phys. Théor. 53 (1990), no. 4, 361-393.

[Kat82] A. Katok, Entropy and closed geodesics, Ergod. Th. E Dynam. Sys. 2 (1982), no. 3-4, 339-365.

[Kif74] Y. Kifer, Small random perturbations of certain smooth dynamical systems, Izv. Akad. Nauk. SSSR; Ser. Mat. 38 (1974), 1091-1115.

[Kif86] Y. Kifer, Ergodic Theory of Random Transformations, Birkhäuser, Basel (1986).

[KY 88] Y. Kifer and Y. Yomdin, Volume growth and topological entropy for random transformations, in Dynamical Systems (College Park, MD, 1986-1987), Springer Lecture Notes Math. 1342 (1988), 361-373. 
[Kun90] H. Kunita, Stochastic flows and stochastic differential equations, Cambridge university press, 1990.

[Led95] F. Ledrappier, Applications of dynamics to compact manifolds of negative curvature, Proceedings of the International Congress of Mathematicians, Vol. 1, 2 (Zürich, 1994), 1195-1202, Birkhäuser, Basel, 1995.

[LS] F. Ledrappier and L. Shu, Entropies for negatively curved manifolds, arXiv preprint arXiv:1905.0274\%.

[LQ95] P.-D. Liu and M. Qian, Smooth ergodic theory of random dynamical systems, Springer Lecture Notes in Math. 1606, Berlin, 1995.

[LS11] P.-D. Liu and L. Shu, Absolute continuity of hyperbolic invariant measures for endomorphisms, Nonlinearity 24 (2011), no. 5, 1596-1611.

[Man79] A. Manning, Topological entropy for geodesic flows, Ann. of Math. (2) 110 (1979), no. 3, $567-573$.

[Man83] R. Mañé, A proof of Pesin's formula, Ergod. Th. E Dynam. Sys. 1 (1981), no. 1, 95-102. Errata to: "A proof of Pesin's formula" Ergod. Th. ES Dynam. Sys. 3 (1983), no. 1, 159-160.

[Ose68] V. I. Oseledec, A multiplicative ergodic theorem. Characteristic Ljapunov, exponents of dynamical systems, Trudy Moskov. Mat. Obšč. 19 (1968), 179-210.

[Rue96] D. Ruelle, Positivity of entropy production in nonequilibrium statistical mechanics, J. Stat. Phys. 85 (1996), no. 1-2, 1-23.

[Shu87] M. Shub, Global stability of dynamical systems, Springer-Verlag, 1987, With the collaboration of Albert Fathi and Rémi Langevin, Translated from the French by Joseph Christy.

[Sul83] D. Sullivan, The Dirichlet problem at infinity for a negatively curved manifold, J. Differential Geometry 18 (1983), no. 4, 723-732.

[Thi92] P. Thieullen, Fibres dynamiques. Entropie et dimension, Ann. Inst. H. Poincaré Anal. Non Linéaire 9 (1992), no. 2, 119-146.

[Yue94] C. B. Yue, Rigidity and dynamics around manifolds of negative curvature, Math. Res. Lett. 1 (1994), no. 2, 123-147.

[Yom87] Y. Yomdin, Volume growth and entropy, Israel J. Math. 57 (1987), no. 3, 285-300. $C^{k}$-resolution of semialgebraic mappings. Addendum to: "Volume growth and entropy", Israel J. Math. $\mathbf{5 7}$ (1987), no. 3, 301-317.

François Ledrappier, Sorbonne Université, UMr 8001, LPSM, Boîte Courrier 158, 4, Place Jussieu, 75252 PARIS CEDEX 05, FrANCE

E-mail address: fledrapp@nd.edu

Lin Shu, Lmam, School of Mathematical Sciences, Peking University, Beijing 100871, PeoPLE's REPUBLIC OF CHINA

E-mail address: 1shu@math.pku.edu.cn 